hep-ph/0505222

WIS/12/05-MAY-DPP, LBNL-57533

\title{
Split Fermions Baryogenesis from the Kobayashi-Maskawa Phase
}

\author{
Gilad Perez* and Tomer Volansky ${ }^{\dagger}$ \\ * Theoretical Physics Group, Ernest Orlando Lawrence Berkeley National Laboratory, \\ Berkeley, University of California, CA 94720 \\ ${ }^{\dagger}$ Weizmann Institute of Science \\ Rehovot, Israel
}

\begin{abstract}
A new scenario of baryogenesis is presented, within the split fermions framework. Our model employs a first order phase transition of the localizer field. The standard model (SM), Kobayashi-Maskawa phase induces a sizable CP asymmetry. The usual suppression of CP violation which arises in the SM baryogenesis is absent due to the existence of order one Yukawa couplings before the fermions are localized in the extra dimension. Models of the above type naturally contain B-L violating operators, allowed by the SM symmetries, which induce the baryon asymmetry. Our mechanism demonstrates the following concept: the flavor puzzle and the SM failure to create the baryon asymmetry are linked and may have a common resolution which does not rely on introduction of new $\mathrm{CP}$ violating sources.
\end{abstract}




\section{Introduction}

It was understood long ago that the SM contains all the ingredients [1] required to produce the baryon asymmetry of the universe (BAU) [2]

$$
\frac{n_{\mathrm{B}}}{s} \sim 7 \times 10^{-11}
$$

where $n_{\mathrm{B}}$ and $s$ are the baryon and entropy densities respectively. Nevertheless the SM fails to explain the observed BAU quantitatively. The lower bound on the Higgs mass [3, 2] implies that the EW phase transition (EWPT) is second order (see e.g. [4, 5] and refs. therein). Thus no departure from thermal equilibrium is obtained. Furthermore, the observed quark flavor parameters are small and hierarchical, posing a puzzle, known as the SM flavor puzzle. A measure of their smallness can be given by the Jarlskog determinant [6], $\mathcal{J}=\operatorname{det}\left[Y_{u} Y_{u}^{\dagger}, Y_{d} Y_{d}^{\dagger}\right]=\mathcal{O}\left(10^{-19}\right)$. In the $\mathrm{SM}$ baryogenesis case, the amount of $\mathrm{CP}$ violation (CPV) produced is suppressed by $\mathcal{J}$ [7, 8, 9, 10, 11. Thus even under the assumption of a first order phase transition (PT), the possibility that the SM can account for the observed BAU is precluded.

It is interesting that the failure of SM baryogenesis is directly related to the SM flavor puzzle. Recently it was demonstrated in [12] that a class of Froggatt-Nielsen [13] models which solve the SM flavor puzzle can also account for the BAU. The main idea of [12] is that within the Froggatt-Nielsen framework, Yukawa couplings can vary with temperature. Thus it is possible that in the early universe the flavor parameters were anarchical, $\mathcal{J} \sim 1$. This implies that CPV arising from the Kobayashi-Maskawa (KM) phase, during the electroweak phase transition, is unsuppressed.

In this work we demonstrate that the five dimensional (5D) split fermions framework can realize the above idea in a similar manner. In our scenario, fermions are localized due to their couplings to an $x_{5}$ dependent VEV of a bulk scalar denoted as the localizer [14, 15, 16. We assume that at the critical temperature, two phases coexist where in one of these phases, the localizer's VEV is zero (the symmetric phase) while in the other it acquires a non-trivial VEV (the broken phase). As in the standard EW baryogenesis, the phase transition occurs through bubble nucleation. Outside the expanding bubble wall (in the unbroken phase) the fermions wave functions are flat [17, 18, 19] and the Yukawa couplings are of order one. The KM phase may therefore be sufficient to explain the observed baryon asymmetry.

Flavor models typically have two distinct phase transitions. The first is related to the scale at which the scalars, which are SM singlets and which control the hierarchy in the flavor sector, 
acquire VEVs. In our model, we denote this phase transition as the localizer phase transition (LPT). The other is the celebrated EWPT. In that context, there are two interesting related possibilities:

(i) The LPT occurs at or below the temperature of the EWPT.

(ii) The LPT occurs at temperatures well above the temperature of the EWPT [19].

Case (i) is very similar to the one discussed in [12. Case (ii) differs from the SM baryogenesis scenario. We show below that it can also naturally avoid all the failures of the SM baryogenesis mechanism and account for the observed BAU. In our framework, we do not require new sources of CPV or new sources which violate baryon number. The related sources within the SM are shown to be sufficient. Furthermore, generically, we expect that in simple split fermions models, option (ii) would be realized as follows: naturally the critical temperature for the LPT, $T_{\mathrm{LPT}}$, will be set by the fundamental scales of the problem $\mathcal{O}(1 / \pi R) \lesssim T_{\mathrm{LPT}} \lesssim M_{*}$ (where $R$ is the radius the extra dimension). Phenomenologically, the inverse radius, $1 / R$ is constrained to be rather high, say above $100 \mathrm{TeV}$, in order to suppress the contributions to various flavor changing processes [20, 21]. We then expect that $T_{\mathrm{LPT}}$ would be of similar order and therefore much above the EW symmetry breaking scale. This implies that the BAU is produced during the LPT, well before the EWPT. Let us briefly describe how Sakharov's conditions are fulfilled in our scenario: $\mathrm{C}$ and $\mathrm{CP}$ violation is of order one in the unbroken phase since all the Yukawa couplings are of order unity. Out of equilibrium is obtained assuming a first order PT. This can be accounted for by, for example, the presence of higher dimension operators in the localizer's potential [19]. Baryon violation occurs since there are non-renormalizable operators which are not suppressed any further by the wave-functions overlapping. It is remarkable that our mechanism goes through even in the most minimal split fermions models a la Kaplan-Tait [21]. We shall see that, despite the fact that in these models the localizer's couplings are CP conserving [19, 22, 23], a sizable baryon asymmetry is obtained.

In section 2 we introduce the split fermions framework and in particular we discuss in some detail the specific model we study to demonstrate our mechanism. In section 3 we describe our mechanism and explain how we derive a semi-quantitative estimation for the resultant asymmetry. In section 4 we show how baryon number violation occurs in our model. This is due to higher dimensional $\mathrm{B}-\mathrm{L}$ violating operators allowed by the SM symmetries. In section 5 we present two complimentary methods to estimate the CP asymmetry due to the interaction with the bubble wall. Many of the detailed calculations relevant to this section are found in 
the appendices. We conclude in section 6

\section{The Framework}

In this part we briefly review some of the relevant features of the split fermions framework. To demonstrate our mechanism we consider a minimal model with a single localizer scalar. To first order the localizer's profile is roughly flat [14, 15] so that at zero temperature such a model is similar to the one in ref [21] with constant odd masses as described below.

We consider one extra dimension compactified on $S^{1} / \mathbb{Z}_{2}$ with a fundamental domain $[0, \pi R]$. The matter content is that of the SM with an additional real scalar "localizer" field $\phi$. The relevant part of the 5-dimensional Lagrangian density is given by

$$
\mathcal{L}_{5}=\bar{\Psi}_{i}\left[i \delta_{i j} \gamma^{M} \partial_{M}-\frac{f_{i j}}{M_{*}^{1 / 2}} \phi\right] \Psi_{j}+\frac{1}{2} \partial_{M} \phi \partial^{M} \phi-V(\phi),
$$

where $M=0, . ., 3,5, \Psi_{i}$ denote SM four-Dirac fermions (which more explicitly are $Q_{i}, u_{i}, d_{i}, L_{i}, e_{i}$

with corresponding couplings $f_{i j}^{Q}, f_{i j}^{u}$ etc., where here and below the representation index is suppressed), $i, j=1, . ., 3$ are the flavor indices, $M_{*} \sim 10 / \pi R$ is the fundamental scale in the theory and we take $\gamma^{5}=-\gamma^{0} \gamma^{1} \gamma^{2} \gamma^{3} . V(\phi)$ is the localizer's potential and a $\phi^{6}$ term is required to obtain a first order PT [19] (see also [5] for a recent study of the 4D case). Due to the 5D Lorentz symmetry the $f$ s are hermitian and therefore can all be brought to a basis in which they are real and diagonal. Thus CP is not broken by the above action. In more complicated (and realistic) models [16] twisting of the fermion wave functions might occur in flavor space [23], providing new CPV sources 19, 22. We shall see below, that even in the limit where these additional sources are switched off, the above framework can still account for the observed BAU.

To avoid the constraints from FCNC [20, 21] $1 / R \gtrsim 100 \mathrm{TeV}$ is required. Note, however, that no upper bound on either $1 / R$ or $M_{*}$ exist. For naturalness we assume that all the dimensionless couplings in the theory are of order one. We emphasize that all our computations below are done at tree level and we assume that quantum effects are sub-dominant.

The orbifold boundary conditions are

$$
\begin{array}{cl}
\phi\left(x^{\mu},-x^{5}\right)=-\phi\left(x^{\mu}, x^{5}\right), & \phi\left(x^{\mu}, x^{5}+\pi R\right)=-\phi\left(x^{\mu}, x^{5}-\pi R\right), \\
\Psi\left(x^{\mu},-x^{5}\right)= \pm \gamma_{5} \Psi\left(x^{\mu}, x^{5}\right), & \Psi\left(x^{\mu}, x^{5}+\pi R\right)= \pm \gamma_{5} \Psi\left(x^{\mu}, x^{5}-\pi R\right),
\end{array}
$$

where the $+(-)$ signs are for $S U(2)$ doublets(singlets). These boundary conditions are intended for two things. For the fermions, these conditions project one of the two chiralities of the zero 
mode, rendering a chiral low-energy theory. For the localizer the conditions impose a non-trivial

$x^{5}$-dependent VEV, which to our purpose is assumed to be a step function. Thus it dynamically plays the role of the odd mass term, $\alpha_{i}$ of ref [21] (for more precise analysis see e.g. [14, 15, 24]). As a consequence of this non-trivial VEV for $\phi$, the fermions zero-modes are localized in the extra dimension,

$$
\Psi_{i}^{(0)}\left(x^{5}\right)=\sqrt{\frac{2 \alpha_{i}}{1-e^{2 \pi \alpha_{i} R}}} \begin{cases}e^{-\alpha_{i} x^{5}} & f_{i i}>0 \\ e^{-\alpha_{i}\left(\pi R-x^{5}\right)} & f_{i i}<0\end{cases}
$$

where $\alpha_{i}=\left|f_{i i} u\right| / M_{*}^{1 / 2}$, and $f_{i i}$ is the eigenvalue of the Yukawa matrix $f_{i j}$ which corresponds to the eigenvector $\Psi_{i}^{(0)}$ (note that $i$ should not be summed in the above). The small overlap between the different zero modes generates the flavor hierarchies. In the model we consider, the hierarchies are realized by taking for the weak doublet fields $f_{i i}>0$ and for the weak singlets $f_{i i}<0$ [21].

\section{Cosmological Setup}

Below we describe the baryon-asymmetry production mechanism and explain how the above framework overcomes the SM difficulties. Our main point here is that, even minimal, split fermions models can efficiently produce baryon number through the LPT. It is the fact that during the LPT, near the expanding localizer bubble wall, two different phases coexist which essentially allows for all of Sakharov's conditions to be realized and lead to a sizable baryon production rate. In the unbroken phase $\mathrm{CPV}$ and $\mathrm{B}-\mathrm{L}$ production rates are unsuppressed while inside the bubble the relevant processes freeze out due to fermion localization.

It is important to identify the time, in the history of the Universe, in which the baryon production occurs. This is directly linked with $T_{\mathrm{LPT}}$, the critical temperature of the LPT. As mentioned above we naturally expect $\mathcal{O}(1 / \pi R) \lesssim T_{\mathrm{LPT}} \lesssim M_{*}$. This is also supported by the phenomenological requirement of the model as follows. The flavor hierarchy is obtained due to the geometrical setup of the fermions in the extra dimension. To account for that, the localizer's mass and other fundamental parameters in its potential, should be of order $\mathcal{O}(10 / \pi R)$ (to have strong enough localization [15, 16]). In this case we then expect that the critical temperature $T_{\mathrm{LPT}}$ would be of similar order. To avoid constraints from processes which induce flavor changing neutral currents the inverse radius is constrained to be rather high, $1 / R \gtrsim 100 \mathrm{TeV}$ [20, 21]. and therefore the LPT occur well before the EWPT,

$$
T_{\mathrm{LPT}} \gg T_{\mathrm{EWPT}}
$$


We shall assume below that $T_{\mathrm{LPT}} \sim 1 / \pi R$. This implies that during the LPT, modes with very high 5D momentum are Boltzmann suppressed. Hence the dynamics of the baryon production can be roughly estimated using our knowledge of $4 \mathrm{D}$ field theory.

Let us discuss in some more detail how Sakharov's conditions are satisfied within our framework.

- CP violation - Outside the bubble wall the quarks wave functions are flat and thus the corresponding Yukawa matrices are naturally sizable and anarchical. The presence of these sizable, flavor breaking, couplings modify the quark thermal masses [25, 8, 9, 11] and imply that CPV is unsuppressed. Since however the LPT occurs before the EWPT it is the fact that the quarks have non-universal, CP conserving, couplings to the localizer which drives the CP asymmetries. This is discussed in more details below.

- B violation - Above the EWPT any B+L asymmetry created is washed out by sphalerons. It is therefore necessary for $\mathrm{B}-\mathrm{L}$ violating interactions to exist and contribute to the asymmetry. It is remarkable that no new ingredients are required to induce B-L number. In fact it was noted a while ago, by Weinberg [26], that non-renormalizable B-L violating terms exist in the SM. These interactions are only mildly suppressed outside the bubble and are frozen in the bubble after fermions localization has occurred. In fact, the $\mathrm{B}-\mathrm{L}$ operators in our case play the same role which is played by the sphaleron processes in the $\mathrm{SM}$ baryogenesis. Consequently, we expect that they would yield baryon production in our case.

- Deviation from thermal equilibrium - First order phase transition can be accounted for by the presence of a $\phi^{6}$ term in the 5D effective localizer's potential [19. This case was recently studied in great details for the SM 4D case [5] where it was indeed found that such terms induce a first order phase transition and consequently deviation from thermal equilibrium.

Let us now briefly describe how the mechanism of baryon production goes through in our model. At $T_{\mathrm{LPT}}$ a phase transition occurs and a bubble of the true vacuum is expanding through space. Just like in the SM baryogenesis, as the wall sweeps through space, particles and antiparticles hit the bubble wall, interacting with the localizer field. From the four dimensional point of view, there is a KK tower of particles and as we show in section [5, the interactions with the wall violate KK number conservation, so an even(odd) incoming KK mode is reflected into an odd(even) KK mode. CP violation implies that the reflection amplitudes for particles 
will be different than the ones for anti-particles resulting in an asymmetry in the corresponding densities near the interface. This asymmetry induces density of a conserved global charge which decays far from the bubble wall due to decoherence effects [27. The induced charge density, in the unbroken phase, biases the $\mathrm{B}-\mathrm{L}$ violating interactions towards production of $\mathrm{B}-\mathrm{L}$ net density [27, 7]. This $\mathrm{B}-\mathrm{L}$ production is shut-off when the expanding bubble wall quickly overtakes the region of non-zero charge and the net $\mathrm{B}-\mathrm{L}$ charge eventually becomes our observed BAU.

An estimate for $n_{B} / s$ may be derived in a similar manner to the one presented in [7, 11]. For simplicity, we assume a $2+1$ dimensional problem with $\left(x^{0}, x^{3}, x^{5}\right)$ spanning space. Thus the computation of the reflection and transmission of particles in the non-compact space becomes a one dimensional problem. Furthermore, we work in the thin wall approximation with low wall velocity, $v_{\mathrm{w}} \simeq 0.1$. Given the above assumptions one obtains [11,

$$
\frac{n_{B}}{s} \simeq-\epsilon_{\mathrm{B}-\mathrm{L}}\left(\frac{15}{2 \pi^{2} g_{*}}\right) \sum_{n, m} \int \frac{d E}{2 \pi T}\left[\left(n_{n+}^{u}-n_{m-}^{u}\right)-\left(n_{n+}^{b}-n_{m-}^{b}\right)\right] \times \Delta_{\mathrm{CP}}^{n m}(E),
$$

where $\epsilon_{\mathrm{B}-\mathrm{L}}$ is a suppression arising from the inefficiency of the B-L violating interactions near the expanding bubble wall and $g_{*} \sim 10^{2}$ is the number of relativistic degrees of freedom. $n_{n \pm}^{u(b)}$ is the Fermi-Dirac distribution boosted to the wall's rest frame, $n_{n \pm}=\left[e^{\left(E-v_{\mathrm{w}} p_{3}^{(n)}\right) / T}+1\right]^{-1}$ for a $\mathrm{n}-\mathrm{KK}$ mode of helicity \pm in the symmetric(broken) phase. Perturbatively, for a small Yukawa coupling to the localizer one can estimate,

$$
\left[\left(n_{n+}^{u}-n_{m-}^{u}\right)-\left(n_{n+}^{b}-n_{m-}^{b}\right)\right] \simeq 2 n_{0}(E)\left[1-n_{0}(E)\right]\left[p_{3}^{(n)}-p_{3}^{(m)}\right] \frac{v_{\mathrm{w}}}{T}
$$

with $p_{3}^{(n)}$ the momentum in the $x^{3}$ direction of the n'th KK mode and $n_{0}(E)=\left(e^{E / T}+1\right)^{-1}$. This is not true in the large coupling limit due to large corrections to the fermion masses, and hence to the momentum. Finally,

$$
\Delta_{\mathrm{CP}}^{n m}(E)=\operatorname{Tr}\left[R_{n m}^{\dagger} R_{n m}^{\dagger}-\bar{R}_{n m}^{\dagger} \bar{R}_{n m}\right]
$$

is the $\mathrm{CP}$ asymmetry related to the reflection coefficients. The quantity $R_{n m}$ is a matrix in flavor space that contains the reflection coefficients. For example, $R_{n m}^{i f}$ is the coefficient for a reflection of an $n$th KK quark state (with, say, helicity + ) and flavor $i$ into an $m$ th KK quark state (with helicity -) and final flavor $f . \bar{R}_{n m}$ corresponds to the $C P$-conjugate processes. In deriving the equation above, we have used the unitarity relation $\sum_{m}\left(R_{n m}^{\dagger} R_{n m}+T_{n m}^{\dagger} T_{n m}\right)=1$ where $T_{n m}$ is the transmission coefficient for the nth KK mode. As we show in Appendix [E this relation obtains corrections of order $p_{3}^{(n)} / T$ which are of order unity. Thus we expect order one corrections to our estimation of $n_{B} / s$. In section $[5.3$ and appendices D and E we 
present a non-perturbative analysis which supports our claim that the above corrections are under control.

A remark is now in order. The $\mathrm{CP}$ asymmetry has contributions from scattering of either the quark doublets or the quark singlets. Each of the three fields $(Q, u, d)$ has an independent coupling to the localizer, and different Yukawa interactions which produce slightly different asymmetries. For simplicity, we calculate below the asymmetry for the quark doublets, however, Yukawa interactions mix between the doublets and singlets and since these interactions are in equilibrium during baryogenesis, the total $\mathrm{CP}$ asymmetry is roughly given by the (weighted) average between the $\mathrm{CP}$ asymmetries of both doublets and singlets.

\section{Baryon Number Violation}

As we explained in section [3 a successful baryogenesis scenario requires a source of B-L violation. Indeed, since the critical temperature $T_{\mathrm{LPT}}$ is well above the $\mathrm{EW}$ symmetry breaking scale, sphaleron processes are fast both inside and outside the bubble. Consequently, the only way to produce baryon number which will not be washed out by sphaleron processes is via $\mathrm{B}-\mathrm{L}$ violating interactions. Furthermore the $\mathrm{B}-\mathrm{L}$ violating processes which are fast in the symmetric phase must be very slow in the broken phase in order to produce the asymmetry. The split fermions framework incorporates such B-L violating processes through non-renormalizable operators. Below we systematically go through the list of these operators, in order to determine which of these is important for our mechanism.

Starting from the lowest dimensional operator, dimension seven in $5 \mathrm{D}$, one finds the celebrated $L^{2} H^{2}$. This operator induces Majorana neutrino masses, but is not directly relevant to baryon production. Such an operator, however, may not only generate too heavy neutrino masses but also washout excess of B-L number and therefore must be suppressed (at least in the broken phase) for our scenario to work. This can be realized using additional extra dimension, a discrete symmetry (see e.g [28] for a recent discussion) or a continuous one [19] giving the lepton doublets some charge so that this term is highly suppressed. Another alternative is if the Higgs is localized away from the lepton doublet. Whatever the mechanism is, we assume that it does not interfere with the baryon production mechanism discussed below.

It was noticed long ago that the symmetries of the SM allow for B-L violating operators [26] 
of dimension 9 and $9 \frac{1}{2}$ in 5D. For completeness, we list these operators

$$
\begin{array}{cll}
\text { Dimension 9: } & \left(\overline{d^{c}} D_{M} d\right)\left(\bar{L} \gamma^{M} Q\right), \quad\left(\bar{L} D_{M} d\right)\left(\overline{d^{c}} \gamma^{M} Q\right), \quad\left(\overline{d^{c}} D_{M} d\right)\left(\bar{e} \gamma^{M} d\right) \\
\text { Dimension 9 } \frac{1}{2}: & \left(\overline{Q^{c}} Q\right)(\bar{L} d) H^{\dagger}, \quad\left(\overline{d^{c}} d\right)(\bar{e} Q) H^{\dagger}, \quad\left(\overline{d^{c}} d\right)(\bar{L} u) H^{\dagger} \\
& \left(\overline{d^{c}} u\right)(\bar{L} d) H^{\dagger}, \quad\left(\overline{d^{c}} d\right)(\bar{L} d) H
\end{array}
$$

where $Q(L)$ stands for a quark(Lepton) doublet, $u, d(e)$ stand for the up and down quark(lepton) singlets, $H$ stands for the Higgs field, $D$ stands for a covariant derivative, c denotes charge conjugation and $M=0 . .3,5$ stands for Lorentz indices. In the above we have suppressed color, flavor and $S U(2)$ indices.

Let us estimate the rate for the dimension $9 \mathrm{~B}-\mathrm{L}$ violating operators of eq. (91). Using naive dimensional analysis (NDA) we estimate the coefficient of the above operators as $(4 \pi)^{2}$ (a $4 \pi$ per interaction assuming the theory becomes strongly coupled at $M_{*}$ ). Thus the relevant part of the Lagrangian is given by

$$
\mathcal{L}=\frac{(4 \pi)^{2}}{M_{*}^{4}}\left(Q D_{M} d d \gamma^{M} \bar{L}+d D_{M} d d \gamma^{M} \bar{l}\right)+\ldots,
$$

where $M_{*}$ is the effective cutoff introduced in section 2, Again, using NDA we estimate the rate for $\mathrm{B}-\mathrm{L}$ violation outside the bubble, $\Gamma_{\mathrm{B}-\mathrm{L}}$ to be roughly

$$
\Gamma_{\mathrm{B}-\mathrm{L}} \sim \frac{(4 \pi)^{4}}{8 \pi}\left(\frac{T}{M_{*}}\right)^{8} T .
$$

In order for the interaction to be in equilibrium we require it to be faster than the expansion rate at the critical temperature,

$$
\Gamma_{\mathrm{B}-\mathrm{L}} \sim \frac{(4 \pi)^{3}}{2} T_{\mathrm{LPT}}\left(\frac{T_{\mathrm{LPT}}}{M_{*}}\right)^{8} \geq \frac{T_{\mathrm{LPT}}^{2}}{M_{\mathrm{Pl}}},
$$

where $M_{\mathrm{Pl}}$ is the reduced Planck mass. Using, $T_{\mathrm{LPT}} \sim 1 / \pi R$, this yields the following condition

$$
\frac{2}{(4 \pi)^{3}}\left(\pi R M_{*}\right)^{7} \frac{M_{*}}{M_{\mathrm{Pl}}} \leq 1
$$

The above condition is easily satisfied over a wide range of $M_{*}$ and $R$. For example for $R M_{*}=\lambda_{C}^{-1}$ as long as $M_{*} \leq 10^{13} \mathrm{GeV}$ the above process is in thermal equilibrium outside the bubble. Interestingly, this yields an upper bound for the theory's cutoff and inverse radius in our model. The exponentially suppressed overlaps between the lepton and quark fields (and between themselves) will ensure that the above processes decouple inside the broken phase.

Finally, we need to estimate the suppression of the baryon number production which is induced by these processes. This suppression occurs due to the fact that the typical time scale 
related to the bubble wall is expected to be of the order of $t_{\mathrm{w}} \sim 4 / T_{\mathrm{LPT}} v_{\mathrm{w}}$ [7, 27]. Thus if $\Gamma_{B-L} t_{\mathrm{w}} \ll 1$ the $\mathrm{B}-\mathrm{L}$ processes are not efficient enough in producing baryon number near the wall, before the corresponding region is overtaken by the bubble. We, therefore, expect that the baryon asymmetry to be suppressed by a factor $\epsilon_{\mathrm{B}-\mathrm{L}}$ given by

$$
\epsilon_{\mathrm{B}-\mathrm{L}} \sim \Delta_{\mathrm{B}-\mathrm{L}} \Gamma_{\mathrm{B}-\mathrm{L}} t_{\mathrm{w}} \sim 4 \frac{(4 \pi)^{3}}{v_{\mathrm{w}}}\left(\frac{1}{\pi R M_{*}}\right)^{8} \sim \frac{1}{v_{\mathrm{w}}}\left(R M_{*}\right)^{-8}
$$

where $\Delta_{\mathrm{B}-\mathrm{L}}=2$ is the $\mathrm{B}-\mathrm{L}$ charge difference induced by a single $\mathrm{B}-\mathrm{L}$ violating process (9).

\section{The CP Asymmetry}

The condition for $\mathrm{CP}$ conservation in the $\mathrm{SM}$ can be formalized by the existence of a matrix $K_{L}$ such that

$$
\begin{aligned}
K_{L}^{\dagger} Y_{u} Y_{u}^{\dagger} K_{L} & =\left(Y_{u} Y_{u}^{\dagger}\right)^{*} \\
K_{L}^{\dagger} Y_{d} Y_{d}^{\dagger} K_{L} & =\left(Y_{d} Y_{d}^{\dagger}\right)^{*}
\end{aligned}
$$

In the five dimensional theory at hand, the couplings with the localizer (see eq. (2)) add the condition (considering for a moment only the quark-doublets) ${ }^{1}$

$$
K_{L}^{\dagger} f_{Q} K_{L}=f_{Q}^{*}
$$

As we now show, CP violation in our cosmological context is found (perturbatively) to be proportional to $\operatorname{Tr}\left[f_{Q}, Y_{u} Y_{u}^{\dagger}+Y_{d} Y_{d}^{\dagger}\right]^{3}$. Generically we expect it to be of order one.

We calculate the CP asymmetry in two different limits as follows:

- Perturbative three-flavor limit, $u / \sqrt{M_{*}} \ll 1 / 2 \pi R$ - where $u$ is the localizer's (roughly constant) VEV. This would allow for an analytical computation of the CP asymmetry within a full three generation framework.

- Non-perturbative single flavor limit, $u / \sqrt{M_{*}} \gtrsim 1 / 2 \pi R$ - an analytic solution is found for the reflection amplitude within a single flavor framework.

The results of our analysis in both limits shows that the CP asymmetry is expected to be sizable. Generically, the parameters of our model lie somewhere between the above limits. The exact

\footnotetext{
${ }^{1}$ In principle this theory contains ten CPV phases [29]. Here we only consider for simplicity the doublet sector which contains three such new phase. As shown below our mechanism is successful even if all the new phases are switched off which can be achieved naturally as we argue in section 6
} 
result is then understood to have order one corrections, but is not expected to be dramatically changed.

Before moving to the actual analysis let us explain how each of the above limits captures different essential ingredients of the dynamics of the interactions with the bubble wall. Such an analysis can, in fact, be useful also in other baryogenesis models beyond our framework (see e.g [12, 19, 30]). In order to explain the low energy values of the Yukawa couplings, it is required from the quark odd masses to be few times larger than $1 / \mathrm{R}$ [21]. In our framework this implies that the localizer's VEV should be roughly $u \sim 10 / \pi R \sqrt{M_{*}}$ [16. On the other hand, as discussed before, we assume that the critical temperature is of order $T_{\mathrm{LPT}} \sim 1 / \pi R$. For the above value of $u$, given that the quarks typical energy is of the order of the critical temperature, the reflection of the quarks from the localizer wall cannot be treated perturbatively. This implies that the non-perturbative treatment can only serve as a rough estimation for the resultant $\mathrm{CP}$ asymmetry. On the other hand the non-perturbative limit can be solved only within a single flavor framework and therefore describes a CP conserving model. It can only be used to estimate the reflection/transmission probability and not the reflection asymmetries.

\subsection{The Effective Dirac Equation}

Considering a $2+1$ dimensional problem, during the phase transition $\phi$ obtains an $x^{5}$ dependent VEV and the bubble of true vacuum is expanding in the $x^{3}$ direction. Hence the relevant part of the Lagrangian takes the form

$$
\mathcal{L}_{5}=i \bar{\Psi}_{i} \gamma^{M} \partial_{M} \Psi_{i}-f_{i j}\left(x^{3}, x^{5}\right) \bar{\Psi}_{i} \Psi_{j}
$$

where within the thin wall approximation,

$$
f_{i j}\left(x^{3}, x^{5}\right)=\left\{\begin{array}{ll}
0 & x^{3} \leq 0 \\
\left(u / M_{*}^{1 / 2}\right) f_{i j} & x^{3}>0
\end{array} .\right.
$$

To demonstrate how the asymmetry is being produced, we consider only the quark doublets, namely $f=f^{Q}$. The computation of the CP asymmetry for the other quarks follow in the same manner and the total asymmetry is obtained through the thermalization of the doublets and the singlets due to Yukawa interactions. Written in matrix form, it is apparent that the spin-up and spin-down parts of the Dirac fermion decouple,

$$
\begin{aligned}
& \mathcal{L}_{5}=\left(\begin{array}{ll}
\Psi_{+}^{1}{ }^{\dagger} & \Psi_{-}^{3}{ }^{\dagger}
\end{array}\right)\left(\begin{array}{cc}
E+i \partial_{3} & -f\left(x^{3}, x^{5}\right)-\partial_{5} \\
-f\left(x^{3}, x^{5}\right)+\partial_{5} & E-i \partial_{3}
\end{array}\right)\left(\begin{array}{l}
\Psi_{+}^{1} \\
\Psi_{-}^{3}
\end{array}\right) \\
&+\left(\begin{array}{ll}
\Psi_{+}^{2 \dagger} & \Psi_{-}^{4}{ }^{\dagger}
\end{array}\right)\left(\begin{array}{cc}
E-i \partial_{3} & -f\left(x^{3}, x^{5}\right)-\partial_{5} \\
-f\left(x^{3}, x^{5}\right)+\partial_{5} & E+i \partial_{3}
\end{array}\right)\left(\begin{array}{l}
\Psi_{+}^{2} \\
\Psi_{-}^{4}
\end{array}\right)
\end{aligned}
$$


where the \pm subscripts stands for the $4 \mathrm{D}$ chirality and we have suppressed flavor indices.

In order to compute the $\mathrm{CP}$ asymmetry, one must take into account fermionic interactions with the plasma. To do so, one introduces the notion of quasiparticles [25, 8, 9, 11], which are fermionic excitations in the plasma. As we review in Appendix $\mathrm{A}$, the effective Dirac equation is found to be

$$
\begin{aligned}
& \left(\begin{array}{cc}
\mathcal{P}+i \partial_{3}+i / 2 l & -\mathcal{F}\left(x^{5}\right) \theta\left(x^{3}\right)-\partial_{5} \\
-\mathcal{F}\left(x^{5}\right) \theta\left(x^{3}\right)+\partial_{5} & \mathcal{P}-i \partial_{3}+i / 2 l
\end{array}\right) \chi\left(x^{3}, x^{5}\right)=0, \\
& \left(\begin{array}{cc}
\mathcal{P}-i \partial_{3}+i / 2 l & -\mathcal{F}\left(x^{5}\right) \theta\left(x^{3}\right)-\partial_{5} \\
-\mathcal{F}\left(x^{5}\right) \theta\left(x^{3}\right)+\partial_{5} & \mathcal{P}+i \partial_{3}+i / 2 l
\end{array}\right) \tilde{\chi}\left(x^{3}, x^{5}\right)=0,
\end{aligned}
$$

where $l$ is the coherence length of the quasiparticles given by $l \simeq 1 / g_{s}^{2} T$ [31, 11]. Thus the quasiparticles are damped as they propagate in space. We also define,

$$
\chi \equiv\left(\begin{array}{l}
\Psi_{+}^{1} \\
\Psi_{-}^{3}
\end{array}\right) ; \quad \tilde{\chi} \equiv\left(\begin{array}{c}
\Psi_{+}^{2} \\
\Psi_{-}^{4}
\end{array}\right)
$$

and

$$
\begin{aligned}
\mathcal{F}\left(x^{5}\right) & \equiv\left(3 u\left(x^{5}\right) / 2 M_{*}^{1 / 2}\right) f \\
\mathcal{P} & \equiv 3(E-\Omega) .
\end{aligned}
$$

Here $\Omega$ denotes the thermal masses of the quasiparticles which are given, to leading order, by 25, 8, 11,

$$
\begin{aligned}
\Omega_{Q}^{2} & =\frac{2 \pi \alpha_{s} T^{2}}{3}+\frac{\pi \alpha_{W} T^{2}}{2}\left(\frac{3}{4}+\frac{\sin ^{2} \theta_{W}}{36}\right)+\frac{T^{2}}{16}\left(Y_{u} Y_{u}^{\dagger}+Y_{d} Y_{d}^{\dagger}\right) \\
\Omega_{u}^{2} & =\frac{2 \pi \alpha_{s} T^{2}}{3}+\frac{2 \pi \alpha_{W} \sin ^{2} \theta_{W} T^{2}}{9}+\frac{T^{2}}{4} Y_{u}^{\dagger} Y_{u} \\
\Omega_{d}^{2} & =\frac{2 \pi \alpha_{s} T^{2}}{3}+\frac{2 \pi \alpha_{W} \sin ^{2} \theta_{W} T^{2}}{9}+\frac{T^{2}}{4} Y_{d}^{\dagger} Y_{d} .
\end{aligned}
$$

Note that here $Y_{u}$ and $Y_{d}$ are order one couplings as opposed to the four dimensional case. Here we have neglected contributions related to the localizer couplings which, although introduce further flavor dependence, do not play a role in our scenario.

We next decompose the fields into KK modes so that the above equations acquire the form similar to the $4 \mathrm{D}$ case. This is done in detail in appendix $\mathrm{A}$. We find,

$$
\begin{aligned}
& \left(\begin{array}{cc}
\mathcal{P}+i \partial_{3}+i / 2 l & -\theta\left(x^{3}\right) \mathcal{F}^{m n}-p_{5}^{(n)} \\
-\theta\left(x^{3}\right) \mathcal{F}^{T m n}-p_{5}^{(n)} & \mathcal{P}-i \partial_{3}+i / 2 l
\end{array}\right) \chi^{(n)}\left(x^{3}\right)=0 \\
& \left(\begin{array}{cc}
\mathcal{P}-i \partial_{3}+i / 2 l & -\theta\left(x^{3}\right) \mathcal{F}^{m n}-p_{5}^{(n)} \\
-\theta\left(x^{3}\right) \mathcal{F}^{T m n}-p_{5}^{(n)} & \mathcal{P}+i \partial_{3}+i / 2 l
\end{array}\right) \tilde{\chi}^{(n)}\left(x^{3}\right)=0 .
\end{aligned}
$$


Here, $p_{5}^{(n)}=n / R$, we have suppressed flavor indices, summation over KK indices is implied and $\mathcal{F}^{n m}$ is given by,

$$
\mathcal{F}_{i j}^{m n}=\left\{\begin{array}{ll}
\frac{6 u}{\pi M_{*}^{1 / 2}} \frac{n}{n^{2}-m^{2}} f_{i j} & m-n \in 2 Z+1 \\
0 & \text { Otherwise }
\end{array} .\right.
$$

We remind the reader that for quark doublets (singlets), $\Psi_{-}^{(0)}\left(\Psi_{+}^{(0)}\right)$ is projected out.

\subsection{A Perturbative Approach}

We now turn to solve eq. (30) perturbatively, assuming $\mathcal{F}^{m n}$ is small. This allows us to find a quantitative estimate for the size of the induced asymmetry. Since the critical temperature is of the order of inverse the 5D radii, it is enough, when calculating the transmission and reflection coefficients, to consider only the first few KK modes. All higher modes are unexcited due to a strong exponential Boltzmann suppression. In practice we shall only consider the zero and the first KK states (note that for each fermion there is a single zero mode and two first KK modes with different helicities).

As is clear from the Green's functions described below, only $\chi$ has an incoming zero-mode. Hence considering $\chi$ and adding a source for the incoming zero-mode, eq. (30) becomes,

$$
\begin{aligned}
& \left(\begin{array}{cc}
\mathcal{P}_{i j}+i \partial_{3}+i / 2 l & -p_{5}^{(n)} \\
-p_{5}^{(n)} & \mathcal{P}_{i j}-i \partial_{3}+i / 2 l
\end{array}\right)\left(\begin{array}{l}
\Psi_{j+}^{1(n)}\left(x^{3}\right) \\
\Psi_{j-}^{3(n)}\left(x^{3}\right)
\end{array}\right)= \\
& \left(\begin{array}{cc}
-i \delta\left(x^{3}\right) \delta_{i j} \delta^{0 m} \delta^{n 0} & \theta\left(x^{3}\right) \mathcal{F}_{i j}^{n m} \\
-\theta\left(x^{3}\right) \mathcal{F}_{i j}^{T n m} & 0
\end{array}\right)\left(\begin{array}{l}
\Psi_{j+}^{1(m)}\left(x^{3}\right) \\
\Psi_{j-}^{3(m)}\left(x^{3}\right)
\end{array}\right) .
\end{aligned}
$$

This equation describes free fermions with mass $p_{5}^{(n)}$ propagating in the unbroken phase, taken to be at $x^{3}<0$. As fermions hit the wall, they interact with the localizer which changes their chirality and partially reflect them back. We treat $\mathcal{F}^{m n}$ perturbatively, describing quasiparticles which reflect back and forth inside the broken phase. Due to the non-diagonal form of $\mathcal{F}^{m n}$ in KK space, an incoming zero-mode is reflected back into an odd KK-mode (see eq. (32)). For the reasons mentioned above we consider only an additional first excited KK mode, and so we seek an expression for the reflection coefficients, describing the scattering of zero-mode into a first $\mathrm{KK}$ mode. In order to be sensitive to $\mathrm{CP}$ violation, we must take into account multiple scattering so as to obtain interference. 
The free Green's function for this problem is constructed in Appendix B We find,

$$
\begin{aligned}
\hat{G}^{(n)}\left(x^{3}-x^{3 \prime}\right)= & -\left(\begin{array}{cc}
\frac{\mathcal{P}+p_{3}^{(n)}}{2 p_{3}^{(n)}} & \frac{p_{5}^{(n)}}{2 p_{3}^{(n)}} \\
\frac{p_{5}^{(n)}}{2 p_{3}^{(n)}} & \frac{\mathcal{P}-p_{3}^{(n)}}{2 p_{3}^{(n)}}
\end{array}\right) \theta\left(x^{3}-x^{3 \prime}\right) e^{i p_{3}^{(n)} x^{3}} \\
& -\left(\begin{array}{cc}
\frac{\mathcal{P}-p_{3}^{(n)}}{2 p_{3}^{(n)}} & \frac{p_{5}^{(n)}}{2 p_{3}^{(n)}} \\
\frac{p_{5}^{(n)}}{2 p_{3}^{(n)}} & \frac{\mathcal{P}+p_{3}^{(n)}}{2 p_{3}^{(n)}}
\end{array}\right) \theta\left(x^{3 \prime}-x^{3}\right) e^{-i p_{3}^{(n)} x^{3}},
\end{aligned}
$$

where $p_{3}^{(n)}=\sqrt{\mathcal{P}^{2}-\left(p_{5}^{(n)}\right)^{2}}+i / 2 l$ is flavor dependent through $\mathcal{P}$ and is KK-dependent though $p_{5}^{(n)}$. Using the Green's function one easily finds the reflection coefficient. We do this in Appendix [C] For now we give the result and note that as opposed to the usual $4 \mathrm{D}$ case where in order to see CP violation one must expand to fifth order in perturbation theory, here CP violation is apparent already at third order. Indeed,

$$
\left.R_{01}=i\left(\begin{array}{l}
G_{12}^{(1)} \mathcal{F} G_{11}^{(0)}\left[1-\mathcal{F} G_{22}^{(1)} \mathcal{F} G_{11}^{(0)}\right. \\
G_{22}^{(1)} \mathcal{F} G_{11}^{(0)}\left[1-\mathcal{F} G_{22}^{(1)} \mathcal{F} G_{11}^{(0)}\right.
\end{array}\right]\right)
$$

where here $\mathcal{F}=\mathcal{F}^{01} g_{s}^{-2} 2 \pi R=\left(12 u R / g_{s}^{2} M_{*}^{1 / 2}\right) f$ and the sub-indices refer to chirality space.

Plugging the above reflection coefficient in the CP-odd expression $\Delta_{\mathrm{CP}}^{n m}$ given in (8) and expanding the Green's function in the flavor dependent part, one finds (see Appendix [C),

$$
\Delta_{\mathrm{CP}}^{01} \propto l^{3} \frac{1}{3} \operatorname{Tr}(\mathcal{F}) \operatorname{tr}\left([\mathcal{F}, \delta \mathcal{P}]^{3}\right)=l^{3} \operatorname{Tr}(\mathcal{F}) \operatorname{det}([\mathcal{F}, \delta \mathcal{P}]) \simeq 10^{-2}
$$

with $\delta \mathcal{P}_{Q}=(T / 32)\left(Y_{u} Y_{u}^{\dagger}+Y_{d} Y_{d}^{\dagger}\right)$ and $\delta \mathcal{P}_{u, d}=(T / 8) Y_{u, d} Y_{u, d}^{\dagger}$. This result, as advocated, has only mild numerical suppressions arising from the loop corrections to the thermal masses.

\subsection{A Non-perturbative Approach}

The reflection coefficients may be calculated in a very different way. Instead of the perturbative approach which is sensitive to the CPV in the theory we can solve a simpler reflection problem semi-analytically. This is important since in order to solve the flavor puzzle we require $f$ of order a few which is beyond the perturbative regime. The problem with the following approach is that the calculation can only be done in a single flavor framework so CP violation is absent. Our attempt here is therefore only to have a quantitative estimate for the magnitude of the reflection coefficients. We then assume that when promoting the problem to accommodate three generations, the reflection coefficients become complex while their magnitudes only change 


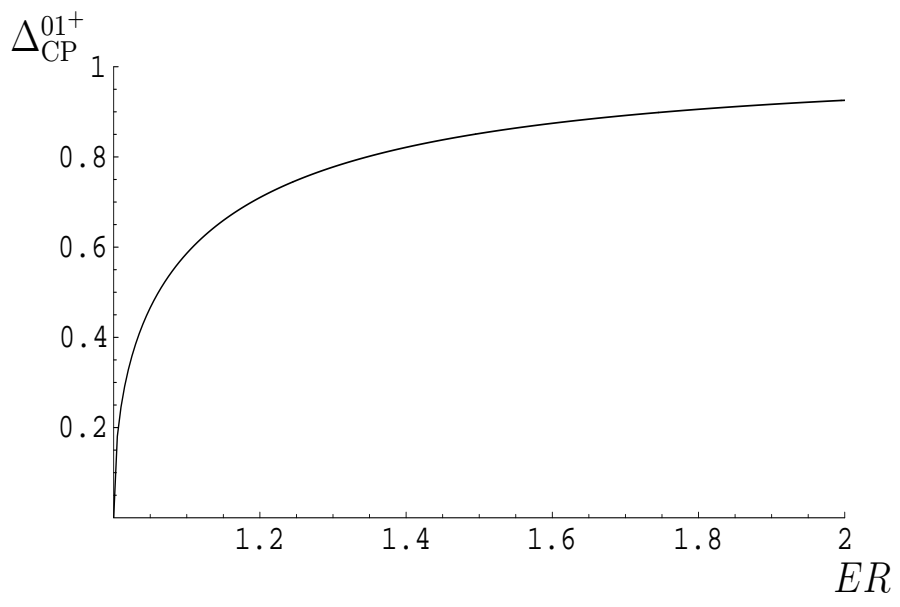

Figure 1: Estimation of $\Delta(E)^{01^{+}}$, the probability of a zero mode to be reflected into a positive helicity state, in a single flavor model for $1<E R<2$.

by order one coefficients. The explicit calculation is done in Appendix $\mathbb{D}$ and here we only summarize our results.

For the calculation we adopt the following strategy. We apply the thin wall approximation and solve the Dirac equation exactly inside and outside the bubble for the whole KK tower. Since the dominant contributions come from the lowest KK modes, we consider only the zeroth and first KK modes as on shell modes (i.e. only these modes will contribute to the reflection and transmission asymmetry). We then match the wave functions of the incoming zero mode and the first KK mode outside the bubble to the outgoing ones inside the bubble. This allows us to extract the reflection coefficients. While this procedure is clear and analytic, the final stage of the actual matching involves the matching of a single state outside the bubble to an infinite tower of (virtual) states inside the bubble which can only be done numerically. We verified our numerical results in several limiting cases as described in the Appendix. Our main result is that a sizable reflection is found over a wide range of energies [19. This is shown in fig. 1which shows the reflection coefficient for an incoming zero mode. It is apparent from the figure, that the reflection coefficients vanish as the energy drops below $1 / R$. This is expected since at such energies only the zero-modes are excited, and thus cannot be reflected by the wall. In our analysis we only consider the first KK states so that it applies only for $w<2 / R$.

As a final remark, we list the correct unitarity relations between the reflection and transmission coefficients, which are calculated in Appendix E

$$
r_{k}\left|R_{01^{+}}\right|^{2}+\left|T_{00}\right|^{2}=1, \quad\left|R_{1^{-} 1^{+}}\right|^{2}+\frac{1}{r_{k}}\left|T_{1^{-} 0}\right|^{2}=1, \quad \frac{1}{r_{k}}\left|R_{1^{+} 0}\right|^{2}+\left|R_{1+1^{-}}\right|^{2}=1,
$$


where $r_{k}=\sqrt{1-\frac{1}{E^{2} R^{2}}}$ and we have explicitly written the helicities of the incoming and outgoing states. As was remarked in section 3 these exact relation would imply that the final expression for $n_{B} / s$ is slightly altered when the realistic values are considered for the model's fundamental parameters. Nevertheless our-order-of-magnitude estimate for the asymmetry is insensitive for such order one effects.

\section{Discussion \& Conclusions}

We begin this part by presenting our final result. In section 4 we showed that, $\epsilon_{\mathrm{B}-\mathrm{L}}$, the suppression from the inefficiency of the $\mathrm{B}-\mathrm{L}$ production near the bubble wall is given by $\epsilon_{\mathrm{B}-\mathrm{L}} \sim 1 /\left[v_{\mathrm{w}}\left(R M_{*}\right)^{8}\right]$. In section 5$]$ we find that, $\Delta_{\mathrm{CP}}^{01}$, the CP asymmetry in the reflection rates is mildly suppressed and is given by $\Delta_{\mathrm{CP}}^{01} \sim 10^{-2}$. Boltzmann suppression and other numerical factors provide another suppression of order $\mathcal{O}\left(10^{-1}\right)$. We therefore estimate the ratio between the baryon asymmetry and entropy to be given by

$$
\frac{n_{B}}{s} \sim \frac{10^{-1}}{g_{*}} \epsilon_{\mathrm{B}-\mathrm{L}} \Delta_{\mathrm{CP}}^{01} \sim 10^{-10} \times\left(\lambda_{C} \times R M_{*}\right)^{-8} .
$$

where $g_{*} \sim 10^{2}$ and $\lambda_{C} \sim 0.22$ is the Cabibbo mixing angle and for comparison with FroggattNielsen type models it is convenient to consider $1 / R M_{*}$ in units of $\lambda_{C}$. This is since in the Froggatt-Nielsen framework $\lambda_{C}$ sets the ratio between the Froggatt-Nielsen scalar VEV and the effective cut-off scale. Thus our estimation for the baryon asymmetry is consistent with the observed value.

The flavor puzzle of the standard model (SM) is related to the fact that most of the flavor parameters are small and hierarchical which hints towards physics beyond the SM. Several kinds of solutions have been suggested to solve the above puzzle. They all require additional fields with new dynamics. Our main message in this work can be stated as follows: In a cosmological context the additional dynamics, which exists in flavor models, naturally incorporates order one Yukawa couplings in the early universe. This could result with a baryon asymmetry produced with the KM phase as the only source of CPV. Thus it is plausible that the flavor puzzle and the SM failure to create the baryon asymmetry are linked and have a common resolution which does not require the presence of new $\mathrm{CP}$ violating sources. This is not to say that no new CP violating sources exist beyond the SM. We merely point out that the KM phase may play an important role in the production of the baryon asymmetry.

In 12 the above concept was introduced and realized in Froggatt-Nielsen type models in 
four dimensions. In this paper we illustrate how a similar behaviour is obtained within a different framework which solves the flavor puzzle, that is the split fermion framework. Assuming a first order phase transition in which a field (the localizer) responsible for the localization obtains a VEV, a net baryon number is produced. CPV occurs through interactions of fermions with the bubble wall. Furthermore non-renormalizable B-L violating interactions, which are allowed by the SM symmetries, induce baryon production outside the bubble wall. These interactions decouple in the broken phase due to fermions localization and thus play a similar role to the sphaleron processes in the SM case.

We argued above that no new sources of $\mathrm{CP}$ violation are required for our mechanism to work. In our model $\mathrm{CP}$ violation was found to be proportional to (at the perturbative level) $\mathcal{J}_{f}=\operatorname{Tr}(f) \operatorname{det}\left[f, Y^{\dagger} Y\right]$ where $f$ and $Y$ denote the Yukawa couplings of the fermions to the localizer and the Higgs respectively. Both $f$ and $Y$ are matrices in flavor space and are expected to be anarchical (we omit the flavor and representation indices for simplicity). Let us demonstrate that indeed one can construct a natural model in which the KM phase is the only $\mathrm{CP}$ violating source. Consider a model in which the two Yukawa matrices are promoted to be fields which transform as bi-fundamentals of the corresponding SM $U(3)_{Q} \times U(3)_{u} \times U(3)_{d}$ flavor group. In the case in which only these two bi-fundamental fields break the flavor group the non-universal part of $f$ will be given, to leading order, by a bilinear combination of the Yukawas. Thus it is clear that any CP violating quantity in the above theory (in particular $\mathcal{J}_{f}$ ) must be proportional to the commutator of the two bi-fundamental fields. Thus the only CP violating source is the KM phase. This should hold even in the presence of quantum correction and therefore technically natural.

In [19] it was demonstrated that realistic split fermion models may induce lepton production from different $\mathrm{CP}$ violating sources [22, namely through twisting of the fermion wave function in flavor space 23]. It is interesting that the effect described above is complimentary to the one induced via twisting and in general both are expected to be present.

Our mechanism requires that the critical temperature should be of the order of the inverse compactification scale, $1 / R$. Phenomenological constraints typically yield $1 / R \gtrsim 100 \mathrm{TeV}$. This implies that our framework allows for a rather low reheating temperature, after inflation, solving the various moduli/gravitino problems. Finally, we note that our model which is based on Kaplan-Tait type models [21, with a constant localizer VEV, is compatible with five dimensional supersymmetric split fermion models (see e.g. [32]). Hence it can easily be supersymmetrized. 


\section{$7 \quad$ Acknowledgments}

The authors thank Yaron Antebi, Guy Engelhard, Guy Raz and Ze'ev Surujon for useful discussions and especially Yuval Grossman, Antonio Masiero and Yossi Nir for useful discussions and comments on the manuscript. GP thanks the Aspen Center for Physics and the Institute of Theoretical Physics, Technion for hospitality while this work was in progress. GP is supported by DOE under contract DE-AC03-76SF00098.

\section{References}

[1] A. D. Sakharov, Pisma Zh. Eksp. Teor. Fiz. 5 (1967) 32 [JETP Lett. 5 (1967 SOPUA,34,392393.1991 UFNAA,161,61-64.1991) 24].

[2] S. Eidelman et al. [Particle Data Group], Phys. Lett. B 592, 1 (2004).

[3] [LEP Collaboration], arXiv:hep-ex/0312023.

[4] K. Kajantie, M. Laine, K. Rummukainen and M. E. Shaposhnikov, Phys. Rev. Lett. 77, 2887 (1996) arXiv:hep-ph/9605288; K. Rummukainen, M. Tsypin, K. Kajantie, M. Laine and M. E. Shaposhnikov, Nucl. Phys. B 532, 283 (1998) arXiv:hep-lat/9805013. F. Csikor, Z. Fodor and J. Heitger, Phys. Rev. Lett. 82, 21 (1999) arXiv:hep-ph/9809291; G. Servant, JHEP 0201, 044 (2002) arXiv:hep-ph/0112209.

[5] C. Grojean, G. Servant and J. D. Wells, Phys. Rev. D 71, 036001 (2005) arXiv:hep-ph/0407019.

[6] C. Jarlskog, Phys. Rev. Lett. 55, 1039 (1985).

[7] A. E. Nelson, D. B. Kaplan and A. G. Cohen, Nucl. Phys. B 373, 453 (1992); A. G. Cohen, D. B. Kaplan and A. E. Nelson, Ann. Rev. Nucl. Part. Sci. 43, 27 (1993) arXiv:hep-ph/9302210.

[8] G. R. Farrar and M. E. Shaposhnikov, Phys. Rev. Lett. 70, 2833 (1993) [Erratum-ibid. 71, 210 (1993)] arXiv:hep-ph/9305274.

[9] G. R. Farrar and M. E. Shaposhnikov, Phys. Rev. D 50, 774 (1994) arXiv:hep-ph/9305275.

[10] M. B. Gavela, P. Hernandez, J. Orloff and O. Pene, Mod. Phys. Lett. A 9, 795 (1994) arXiv:hep-ph/9312215; M. B. Gavela, P. Hernandez, J. Orloff, O. Pene and C. Quimbay, Nucl. Phys. B 430, 382 (1994) arXiv:hep-ph/9406289. 
[11] P. Huet and E. Sather, Phys. Rev. D 51, 379 (1995) arXiv:hep-ph/9404302.

[12] M. Berkooz, Y. Nir and T. Volansky, Phys. Rev. Lett. 93, 051301 (2004) arXiv:hep-ph/0401012.

[13] C. D. Froggatt and H. B. Nielsen, Nucl. Phys. B 147, 277 (1979).

[14] H. Georgi, A. K. Grant and G. Hailu, Phys. Rev. D 63, 064027 (2001) arXiv:hep-ph/0007350.

[15] G. Perez, Phys. Rev. D 67, 013009 (2003) arXiv:hep-ph/0208102;

[16] Y. Grossman and G. Perez, Phys. Rev. D 67, 015011 (2003) arXiv:hep-ph/0210053.

[17] A. Masiero, M. Peloso, L. Sorbo and R. Tabbash, Phys. Rev. D 62, 063515 (2000) arXiv:hep-ph/0003312.

[18] D. J. H. Chung and T. Dent, Phys. Rev. D 66, 023501 (2002) arXiv:hep-ph/0112360.

[19] Y. Nagatani and G. Perez, JHEP 0502, 068 (2005) arXiv:hep-ph/0401070.

[20] A. Delgado, A. Pomarol and M. Quiros, JHEP 0001, 030 (2000) hep-ph/9911252; B. Lillie and J. L. Hewett, Phys. Rev. D 68, 116002 (2003) hep-ph/0306193;

[21] D. E. Kaplan and T. M. P. Tait, JHEP 0111, 051 (2001) arXiv:hep-ph/0110126.

[22] R. Harnik, G. Perez, M. D. Schwartz and Y. Shirman, JHEP 0503, 068 (2005) arXiv:hep-ph/0411132.

[23] Y. Grossman, R. Harnik, G. Perez, M. D. Schwartz and Z. Surujon, Phys. Rev. D 71, 056007 (2005) arXiv:hep-ph/0407260.

[24] B. Grzadkowski and M. Toharia, Nucl. Phys. B 686, 165 (2004) arXiv:hep-ph/0401108.

[25] H. A. Weldon, Phys. Rev. D 26, 1394 (1982).

[26] S. Weinberg, Phys. Rev. D 22, 1694 (1980).

[27] A. E. Nelson, D. B. Kaplan and A. G. Cohen, Phys. Lett. B 294, 57 (1992) arXiv:hep-ph/9206214. S. Y. Khlebnikov, Phys. Lett. B 300, 376 (1993).

[28] H. Davoudiasl, R. Kitano, G. D. Kribs and H. Murayama, arXiv:hep-ph/0502176

[29] K. Agashe, G. Perez and A. Soni, Phys. Rev. D 71, 016002 (2005) arXiv:hep-ph/0408134. 
[30] L. J. Hall, H. Murayama and G. Perez, arXiv:hep-ph/0504248.

[31] E. Braaten and R. D. Pisarski, Phys. Rev. D 46, 1829 (1992).

[32] D. E. Kaplan and T. M. P. Tait, JHEP 0006, 020 (2000) arXiv:hep-ph/0004200; E. A. Mirabelli and M. E. Peskin, Phys. Rev. D 58, 065002 (1998) arXiv:hep-th/9712214; N. Arkani-Hamed, T. Gregoire and J. Wacker, JHEP 0203, 055 (2002) arXiv:hep-th/0101233; N. Arkani-Hamed, L. J. Hall, D. R. Smith and N. Weiner, Phys. Rev. D 63, 056003 (2001) arXiv:hep-ph/9911421; H. Abe, K. Choi and I. W. Kim, JHEP 0409, 001 (2004) arXiv:hep-th/0405100.

\section{APPENDIX}

\section{A The Effective Dirac Equation and KK Decomposition}

As explained in [11, the dispersion relation for quasiparticles may be found by looking for the poles of the full propagator, including the self-eneries. Taking thermal self-energy of the form,

$$
\Sigma(E, \vec{p})=\gamma^{0} a(E, p)-b(E, p) \vec{\gamma} \cdot \vec{p}
$$

one is then looking for a solution to

$$
\operatorname{det}\left[S_{0}^{-1}-\Sigma(E, \vec{p})\right]=0
$$

with $S_{0}^{-1}=\gamma^{0} E-\vec{\gamma} \cdot \vec{p}$. Considering the $2+1$ problem at hand, the solution to the above is

$$
E=a(E, p) \pm[1-b(E, p)] \sqrt{p_{3}^{2}+p_{5}^{2}}
$$

In the four dimensional case, the self-energy can be linearized as

$$
\Sigma(E, \vec{p}) \simeq \gamma^{0}(2 \Omega-i / 3 l-E)-\vec{\gamma} \cdot \vec{p} / 3
$$

where as mentioned in section [5.1] $\Omega$ denotes the thermal mass of the quasiparticle and $l$ is the coherence length of the quasiparticles given by $l=1.1 / g_{s}^{2} T$ [31, 11]. This is of course also true in the broken phase where the theory is effectively $4 \mathrm{D}$. In the symmetric phase, the theory is effectively $5 \mathrm{D}$ and thus we expect corrections to the above. Nevertheless, at the temperature of phase transition, the 5D linearized self-energies are expected to be affected only by a few 
higher KK states and are thus expected, at zeroth order, to admit the same behaviour. With the above, one obtains the dispersion relation,

$$
E=\Omega-\frac{i}{6 l} \pm \frac{1}{3} \sqrt{p_{3}^{2}+p_{5}^{2}}
$$

The effective free Dirac equation is therefore obtained by taking $\gamma^{\mu} p_{\mu} \rightarrow \gamma^{\mu} p_{\mu}-\Sigma$ which has the effect of taking $E \rightarrow 2(E-\Omega+i / 6 l)$ and $p \rightarrow 2 p / 3$. Thus, plugging these in eq. (20) and massaging a little one finds the effective Dirac equation given in (22), (23).

Next we KK decompose the Dirac fermions. The KK reduction of the wave function is,

$$
\Psi_{i \pm}\left(x^{3}, x^{5}\right)=\sum_{n=0}^{\infty} u_{i n}^{ \pm}\left(x^{5}\right) \Psi_{i \pm}^{(n)}\left(x^{3}\right),
$$

where the \pm are the two chiralities of the Dirac fermion, and $u_{i n}^{ \pm}=a_{i n}^{ \pm} e^{i p_{5} x^{5}}+b_{i n}^{ \pm} e^{-i p_{5} x^{5}}$. The boundary conditions for $u_{i n}^{ \pm}$are given by (again, considering, for example, the quark doublets),

$$
\begin{aligned}
u_{i n}^{ \pm}\left(x^{5}=0^{+}, \pi R^{-}\right) & = \pm u_{i n}^{ \pm}\left(x^{5}=0^{-}, \pi R^{+}\right), \\
\left.\partial_{5} u_{i n}^{+}\right|_{x^{5}=0^{+}, \pi R^{-}} & =f_{i j}\left(x^{5}=0^{+}, \pi R^{-}\right) u_{j n}^{+}\left(x^{5}=0^{+}, \pi R^{-}\right), \\
\left.\partial_{5} u_{i n}^{-}\right|_{x^{5}=0^{+}, \pi R^{-}} & =\left.\partial_{5} u_{i n}^{-}\right|_{x^{5}=0^{-}, \pi R^{+}} .
\end{aligned}
$$

Hence in the unbroken phase,

$$
u_{i n}^{+}=\left(\frac{2}{\pi R}\right)^{1 / 2} \cos \left(\frac{n x^{5}}{R}\right), \quad u_{i n}^{-}=\left(\frac{2}{\pi R}\right)^{1 / 2} \sin \left(\frac{n x^{5}}{R}\right) .
$$

It is important to note that under this decomposition, the thermal masses are KK diagonal. To see this simply note that we assume the Higgs field is $x^{5}$ independent. Thus the Yukawa interactions decompose into

$$
Y_{i j} H \bar{Q} d \longrightarrow Y_{i j} H \sum_{n}\left(\bar{Q}_{+}^{(n)} d_{-}^{(n)}+\bar{Q}_{-}^{(n)} d_{+}^{(n)}\right) .
$$

Here we replaced (temporarily) $\Psi$ by $Q$ and $d$ to stress that the interaction is between two distinct fields. Note that $Q$ and $d$ have orbifold boundary conditions with opposite sign. The above in particular means that $\Omega_{ \pm}^{(n)}$ are independent of $n$. Moreover, $\Omega_{+}=\Omega_{-}$.

On the other hand, the interactions with the localizer are not KK diagonal. We have,

$$
f_{i j}\left(x^{3}, x^{5}\right) \bar{\Psi}_{i} \Psi_{j} \longrightarrow \theta\left(x^{3}\right) \sum_{n m} \frac{2}{3}\left(\mathcal{F}_{i j}^{m n} \bar{\Psi}_{i+}^{(m)} \Psi_{j-}^{(n)}+\mathcal{F}_{i j}^{T m n} \bar{\Psi}_{i-}^{(m)} \Psi_{j+}^{(n)}\right),
$$

with $\mathcal{F}$ given in eqs. (25), (32). In the above, the transpose is with respect to KK number. Note further, that due to the complex conjugate term in the Lagrangian, $\mathcal{F}$ can be taken to be Hermitian with respect to flavor. 


\section{B Computation of the Green's Function}

To construct the (free) Green's functions we solve:

$$
\begin{aligned}
& \left(\begin{array}{cc}
\left(\mathcal{P}_{+}\right)_{i j}+i \partial_{3} & -p_{5}^{(n)} \\
-p_{5}^{(n)} & \left(\mathcal{P}_{-}\right)_{i j}-i \partial_{3}
\end{array}\right) \hat{G}_{j k}^{(n)}\left(x^{3}-x^{3 \prime}\right)=i \delta\left(x^{3}-x^{3 \prime}\right) \delta_{i k} \\
& \left(\begin{array}{cc}
\left(\mathcal{P}_{+}\right)_{i j}-i \partial_{3} & -p_{5}^{(n)} \\
-p_{5}^{(n)} & \left(\mathcal{P}_{-}\right)_{i j}+i \partial_{3}
\end{array}\right) \hat{\tilde{G}}_{j k}^{(n)}\left(x^{3}-x^{3 \prime}\right)=i \delta\left(x^{3}-x^{3 \prime}\right) \delta_{i k}
\end{aligned}
$$

where $\hat{G}_{j k}^{(n)}\left(x^{3}-x^{3 \prime}\right)$ is a $2 \times 2$ matrix. The solution is found by a double Fourier transform. Defining,

$$
\begin{aligned}
& \hat{G}_{j k}^{(n)}\left(x^{3}-x^{3 \prime}\right)=\int d k e^{i k\left(x^{3}-x^{3 \prime}\right)} \hat{G}_{j k}^{(n)}(k) \\
& \hat{\tilde{G}}_{j k}^{(n)}\left(x^{3}-x^{3 \prime}\right)=\int d k e^{i k\left(x^{3}-x^{3 \prime}\right)} \hat{\tilde{G}}_{j k}^{(n)}(k)
\end{aligned}
$$

we obtain (using $\mathcal{P}_{+}=\mathcal{P}_{-} \equiv \mathcal{P}$ ),

$$
\begin{aligned}
& \hat{G}_{j k}^{(n)}(k)=2 \pi i\left(\begin{array}{cc}
\mathcal{P}-k & -p_{5}^{(n)} \\
-p_{5}^{(n)} & \mathcal{P}+k
\end{array}\right)_{j k}^{-1}=-2 \pi i\left(\begin{array}{ll}
\frac{k+\mathcal{P}}{k^{2}-\left(p_{3}^{(n)}\right)^{2}} & \frac{p_{5}^{(n)}}{k^{2}-\left(p_{3}^{(n)}\right)^{2}} \\
\frac{p_{5}^{(n)}}{k^{2}-\left(p_{3}^{(n)}\right)^{2}} & \frac{-k+\mathcal{P}}{k^{2}-\left(p_{3}^{(n)}\right)^{2}}
\end{array}\right)_{j k} \\
& \tilde{\hat{G}}_{j k}^{(n)}(k)=2 \pi i\left(\begin{array}{cc}
\mathcal{P}+k & -p_{5}^{(n)} \\
-p_{5}^{(n)} & \mathcal{P}-k
\end{array}\right)_{j k}^{-1}=-2 \pi i\left(\begin{array}{ll}
\frac{-k+\mathcal{P}}{k^{2}-\left(p_{3}^{(n)}\right)^{2}} & \frac{p_{5}^{(n)}}{k^{2}-\left(p_{3}^{(n)}\right)^{2}} \\
\frac{p_{5}^{(n)}}{k^{2}-\left(p_{3}^{(n)}\right)^{2}} & \frac{k+\mathcal{P}}{k^{2}-\left(p_{3}^{(n)}\right)^{2}}
\end{array}\right)_{j k},
\end{aligned}
$$

where $\left(p_{3}^{(n)}\right)^{2}=\mathcal{P}^{2}-\left(p_{5}^{(n)}\right)^{2}$. This is precisely the Green's function of a massive fermion, $\left(\gamma_{\mu} p^{\mu}+m\right) /\left(p^{2}-m^{2}\right)$ with $m=p_{5}^{(n)}$. The inverse Fourier transform depends on the contour chosen for the integral which is equivalent to choosing a boundary condition. There are four different contours going above or below each of the two poles $k= \pm p_{3}^{(n)}$. For reasons to be understood below, we choose the contour which goes above $k=-p_{3}^{(n)}$ and below $k=+p_{3}^{(n)}$. 
After a Cauchy integral one obtains,

$$
\begin{aligned}
& \hat{G}_{j k}^{(n)}\left(x^{3}-x^{3 \prime}\right)=-\left(\begin{array}{cc}
\frac{\mathcal{P}+p_{3}^{(n)}}{2 p_{3}^{(n)}} & \frac{p_{5}^{(n)}}{2 p_{3}^{(n)}} \\
\frac{p_{5}^{(n)}}{2 p_{3}^{(n)}} & \frac{\mathcal{P}-p_{3}^{(n)}}{2 p_{3}^{(n)}}
\end{array}\right)_{j k} \theta\left(x^{3}-x^{3 \prime}\right) e^{i p_{3}^{(n)} x^{3}} \\
& -\left(\begin{array}{cc}
\frac{\mathcal{P}-p_{3}^{(n)}}{2 p_{3}^{(n)}} & \frac{p_{5}^{(n)}}{2 p_{3}^{(n)}} \\
\frac{p_{5}^{(n)}}{2 p_{3}^{(n)}} & \frac{\mathcal{P}+p_{3}^{(n)}}{2 p_{3}^{(n)}}
\end{array}\right)_{j k} \theta\left(x^{3 \prime}-x^{3}\right) e^{-i p_{3}^{(n)} x^{3}} \\
& \tilde{\hat{G}}_{j k}^{(n)}\left(x^{3}-x^{3 \prime}\right)=-\left(\begin{array}{cc}
\frac{\mathcal{P}-p_{3}^{(n)}}{2 p_{3}^{(n)}} & \frac{p_{5}^{(n)}}{2 p_{3}^{(n)}} \\
\frac{p_{5}^{(n)}}{2 p_{3}^{(n)}} & \frac{\mathcal{P}+p_{3}^{(n)}}{2 p_{3}^{(n)}}
\end{array}\right)_{j k} \theta\left(x^{3}-x^{3 \prime}\right) e^{i p_{3}^{(n)} x^{3}} \\
& -\left(\begin{array}{ll}
\frac{\mathcal{P}+p_{3}^{(n)}}{2 p_{3}^{(n)}} & \frac{p_{5}^{(n)}}{2 p_{3}^{(n)}} \\
\frac{p_{5}^{(n)}}{2 p_{3}^{(n)}} & \frac{\mathcal{P}-p_{3}^{(n)}}{2 p_{3}^{(n)}}
\end{array}\right)_{j k} \theta\left(x^{3 \prime}-x^{3}\right) e^{-i p_{3}^{(n)} x^{3}} .
\end{aligned}
$$

The above matrices can be diagonalized. One finds one left-moving mode and one rightmoving mode. The diagonal Green's functions are given by

$$
\begin{aligned}
& \hat{G}_{j k}^{(n)}\left(x^{3}-x^{3 \prime}\right)=-\left(\begin{array}{cc}
\frac{\mathcal{P}}{p_{3}^{(n)}} \theta\left(x^{3}-x^{3 \prime}\right) e^{i p_{3}^{(n)} x^{3}} & 0 \\
0 & \frac{\mathcal{P}}{p_{3}^{(n)}} \theta\left(x^{3 \prime}-x^{3}\right) e^{-i p_{3}^{(n)} x^{3}}
\end{array}\right)_{j k}, \\
& \tilde{\hat{G}}_{j k}^{(n)}\left(x^{3}-x^{3 \prime}\right)=-\left(\begin{array}{cc}
\frac{\mathcal{P}}{p_{3}^{(n)}} \theta\left(x^{3 \prime}-x^{3}\right) e^{-i p_{3}^{(n)} x^{3}} & 0 \\
0 & \frac{\mathcal{P}}{p_{3}^{(n)}} \theta\left(x^{3}-x^{3 \prime}\right) e^{i p_{3}^{(n)} x^{3}}
\end{array}\right)_{j k}
\end{aligned}
$$

and are obtained through a change of basis with the diagonalizing matrices,

$$
\begin{aligned}
& U^{(n)}=\theta\left(x^{3}-x^{3 \prime}\right)\left(\begin{array}{cc}
p_{3}^{(n)}+\mathcal{P} & \frac{p_{3}^{(n)}-\mathcal{P}}{p_{5}^{(n)}} \\
p_{5}^{(n)} & 1
\end{array}\right)+\theta\left(x^{3 \prime}-x^{3}\right)\left(\begin{array}{cc}
-\left(p_{3}^{(n)}+\mathcal{P}\right) & \frac{p_{3}^{(n)}-\mathcal{P}}{p_{5}^{(n)}} \\
p_{5}^{(n)} & -1
\end{array}\right), \\
& \tilde{U}^{(n)}=\theta\left(x^{3 \prime}-x^{3}\right)\left(\begin{array}{cc}
p_{3}^{(n)}+\mathcal{P} & \frac{p_{3}^{(n)}-\mathcal{P}}{p_{5}^{(n)}} \\
p_{5}^{(n)} & 1
\end{array}\right)+\theta\left(x^{3}-x^{3 \prime}\right)\left(\begin{array}{cc}
-\left(p_{3}^{(n)}+\mathcal{P}\right) & \frac{p_{3}^{(n)}-\mathcal{P}}{p_{5}^{(n)}} \\
p_{5}^{(n)} & -1
\end{array}\right) .
\end{aligned}
$$

Note that with this choice there is a well defined $p_{5} \rightarrow 0$ limit, but the matrices are not orthogonal. One can choose an orthogonal matrix by normalizing the eigenverctors. Note also, that there is only an incoming (outgoing) zero-mode for $\chi(\tilde{\chi})$ since there is no $\chi_{-}^{(0)}, \tilde{\chi}_{-}^{(0)}$. Finally, it is clear that the Green's functions fulfill,

$$
G_{+}(-\infty)=G_{-}(+\infty)=0
$$




\section{A Perturbative Computation of the CP Asymmetry}

Our goal is a perturbative solution of the reflection coefficients for the scattering described by eq. (33). As we argued in section 5.2. the lowest order $s u(3)$-invariant expression is of the form $\operatorname{Tr}\left(A^{3}\right)=3 \operatorname{det}(A)$ for $A \in s u(3)$. Thus the first term to contribute to the asymmetry, $\Delta_{\mathrm{CP}} \sim R_{01}^{\dagger} R_{01}$, is of form $\operatorname{Tr}\left(\left[\mathcal{F}, Y^{\dagger} Y\right]^{3}\right)$ and can therefore arise from fourth order in $\mathcal{F}$. The reflection coefficients, should therefore be calculated to third order in $\mathcal{F}$.

Using the Green's functions, one transforms eq. (33) into an intergral one,

$$
\begin{aligned}
\left(\begin{array}{c}
\Psi_{i+}^{1(n)}\left(x^{3}\right) \\
\Psi_{i-}^{3(n)}\left(x^{3}\right)
\end{array}\right)= & \left(\begin{array}{c}
-i G_{+}^{(0)}\left(x^{3}\right) \Psi_{+i}^{1(0)}(0) \delta^{n 0} \\
0
\end{array}\right)+ \\
& \int d x^{3 \prime} \theta\left(x^{3 \prime}\right) \hat{G}^{(n)}\left(x^{3}-x^{3 \prime}\right)\left(\begin{array}{cc}
0 & \mathcal{F}^{n m} \\
-\mathcal{F}^{T n m} & 0
\end{array}\right)\left(\begin{array}{c}
\Psi_{k-}^{4(m)}\left(x^{3 \prime}\right) \\
\Psi_{k+}^{2(m)}\left(x^{3 \prime}\right)
\end{array}\right),
\end{aligned}
$$

where we suppressed the flavor indices.

The solution is found iteratively,

$$
\begin{aligned}
& \left(\begin{array}{c}
\Psi_{i+}^{1(n)}\left(x^{3}\right) \\
\Psi_{i-}^{3(n)}\left(x^{3}\right)
\end{array}\right)^{0}=\left(\begin{array}{c}
-i G_{11}^{(0)}\left(x^{3}\right) \Psi_{+i}^{1(0)}(0) \delta^{n 0} \\
0
\end{array}\right) \\
& \left(\begin{array}{c}
\Psi_{i+}^{1(n)}\left(x^{3}\right) \\
\Psi_{i-}^{3(n)}\left(x^{3}\right)
\end{array}\right)^{1}=\int d x^{3 \prime} \theta\left(x^{3 \prime}\right) \hat{G}^{(n)}\left(x^{3}-x^{3 \prime}\right)\left(\begin{array}{cc}
0 & \mathcal{F}_{j k}^{n 0} \\
-\mathcal{F}_{j k}^{T n 0} & 0
\end{array}\right)\left(\begin{array}{c}
-i G_{11}^{(0)}\left(x^{3 \prime}\right) \Psi_{+i}^{1(0)}(0) \\
0
\end{array}\right) \\
& \left(\begin{array}{l}
\Psi_{i+}^{1(n)}\left(x^{3}\right) \\
\Psi_{i-}^{3(n)}\left(x^{3}\right)
\end{array}\right)^{2}=\int d x^{3 \prime} d x^{3 \prime \prime} \theta\left(x^{3 \prime}\right) \theta\left(x^{3 \prime \prime}\right) \hat{G}^{(n)}\left(x^{3}-x^{3 \prime}\right)\left(\begin{array}{cc}
0 & \mathcal{F}_{j k}^{n m} \\
-\mathcal{F}_{j k}^{T n m} & 0
\end{array}\right) \hat{G}^{(m)}\left(x^{3 \prime}-x^{3 \prime \prime}\right) \\
& \left(\begin{array}{cc}
0 & \mathcal{F}_{j k}^{m 0} \\
-\mathcal{F}_{j k}^{T m 0} & 0
\end{array}\right)\left(\begin{array}{c}
-i G_{11}^{(0)}\left(x^{3 \prime \prime}\right) \Psi_{+i}^{1(0)}(0) \\
0
\end{array}\right) \\
& \left(\begin{array}{l}
\Psi_{i+}^{1(n)}\left(x^{3}\right) \\
\Psi_{i-}^{3(n)}\left(x^{3}\right)
\end{array}\right)^{3}=\int d x^{3 \prime} d x^{3 \prime \prime} d x^{3 \prime \prime \prime} \theta\left(x^{3 \prime}\right) \theta\left(x^{3 \prime \prime}\right) \theta\left(x^{3 \prime \prime \prime}\right) \hat{G}^{(n)}\left(x^{3}-x^{3 \prime}\right)\left(\begin{array}{cc}
0 & \mathcal{F}_{j k}^{n m} \\
-\mathcal{F}_{j k}^{T n m} & 0
\end{array}\right) \\
& \hat{G}^{(m)}\left(x^{3 \prime}-x^{3 \prime \prime}\right)\left(\begin{array}{cc}
0 & \mathcal{F}_{j k}^{m l} \\
-\mathcal{F}_{j k}^{T m l} & 0
\end{array}\right) \hat{G}^{(l)}\left(x^{3 \prime \prime}-x^{3 \prime \prime \prime}\right)\left(\begin{array}{cc}
0 & \mathcal{F}_{j k}^{l 0} \\
-\mathcal{F}_{j k}^{T l 0} & 0
\end{array}\right) \\
& \left(\begin{array}{c}
-i G_{11}^{(0)}\left(x^{3 \prime \prime \prime}\right) \Psi_{+i}^{1(0)}(0) \\
0
\end{array}\right) .
\end{aligned}
$$

Note that as opposed to the $4 \mathrm{D}$ case, here, in general, all orders contribute to the reflection coefficients due to the non-diagonal form of the Green's functions. Nevertheless, the special form of $\mathcal{F}^{m n}$, eq. (36), permits only odd(even) non-vanishing orders in the expansion for 
an odd(even) outgoing KK mode. Even with this simplification, the general expressions for the reflection coefficients are very awkward. To simplify, we consider only the zeroth and first KK modes. As explained in section 5.2, this is justified since these two modes have the lowest Boltzman suppression factors. Furthermore, numerically, $\mathcal{F}^{n m}$ is larger for $n, m=0,1$. Focusing on these KK modes, we see that the integrals above are highly damped for $x^{3} / l \gg 1$ due to decoherence. We thus estimate them by replacing $x^{3}$ with the decoherence length $l=g_{s}^{-2} 2 \pi R$. Doing so results with eq. (35).

The only flavor dependence in the Green's functions comes from $Y^{\dagger} Y$ in $\mathcal{P}$ (by $Y^{\dagger} Y$ we mean $Y_{u}^{\dagger} Y_{u}+Y_{d}^{\dagger} Y_{d}$ for the $S U(2)$ doublets and $Y_{u(d)} Y_{u(d)}^{\dagger}$ for the singlets). Using $\mathcal{P} \simeq \mathcal{P}_{d}+\delta \mathcal{P}$ and $p_{3}^{(n)}=\sqrt{\left(\mathcal{P}_{d}+\delta \mathcal{P}\right)^{2}-p_{5}^{(n)}}+i / 2 l$ with $\delta \mathcal{P} \propto Y^{\dagger} Y$ we expand

$$
G_{i j}^{(n)}=G_{i j}^{(n) \operatorname{diag}}\left(1+\sum_{k=1}^{\infty} C_{i j, k}^{(n)}(l \delta \mathcal{P})^{k}\right)
$$

where, $G_{i j}^{(n) d i a g}$ is the flavor diagonal Green's function obtained by setting $Y^{\dagger} Y$ to zero, and for the sake of brevity, we denote the flavor independent coefficients by $C_{i j, k}^{(n)}$. Thus for example, $C_{11, k}^{(0)}=i^{k} / k$ ! etc.

Below we choose the flavor basis such that $\mathcal{F}$ is diagonal. Note that the diagonalizing matrix does not contain (by definition) any CP phase, namely, $\mathcal{F}$ is real. So to order $\mathcal{F}^{4}$,

$$
\begin{aligned}
R_{01}^{\dagger} R_{01} & =G_{11}^{(0) \dagger} \mathcal{F}\left(G_{12}^{(1) \dagger} G_{12}^{(1)}+G_{22}^{(1) \dagger} G_{22}^{(1)}\right) \mathcal{F} G_{11}^{(0)} \mathcal{F} G_{22}^{(1)} \mathcal{F} G_{11}^{(0)} \\
& +G_{11}^{(0) \dagger} \mathcal{F} G_{22}^{(1) \dagger} \mathcal{F} G_{11}^{(0) \dagger} \mathcal{F}\left(G_{12}^{(1) \dagger} G_{12}^{(1)}+G_{22}^{(1) \dagger} G_{22}^{(1)}\right) \mathcal{F} G_{11}^{(0)}
\end{aligned}
$$

Each term in eq. (71) contributes to the CP asymmetry seperately. In expanding the Green's functions, the first term to contribute is of order $\delta \mathcal{P}^{3}$. Using the facts that $\delta \mathcal{P}$ is hermitian and that $\bar{R}_{01}$ is obtained from $R_{01}$ by replacing $\delta \mathcal{P}$ with $\delta \mathcal{P}^{T}$ we find, as an example, for the first term in the first line,

$$
\begin{aligned}
\Delta_{\mathrm{CP}} & \propto\left[C_{11,1}^{(0) *}\left(C_{11,1}^{(0)} C_{12,1}^{(1)}+C_{11,1}^{(0)} C_{12,1}^{(1) *}-C_{12,1}^{(1) *} C_{12,1}^{(1)}-C_{12,2}^{(1)}-C_{12,2}^{(1) *}\right)\right. \\
+ & \left.C_{11,2}^{(0) *}\left(C_{12,1}^{(1)}+C_{12,1}^{(1)}\right)\right] \operatorname{Tr}\left(\delta \mathcal{P}^{2} \mathcal{F} \delta \mathcal{P} \mathcal{F}^{3}-\delta \mathcal{P}^{2} \mathcal{F}^{3} \delta \mathcal{P} \mathcal{F}\right) \\
& \propto \operatorname{Tr}\left(\mathcal{F}[\mathcal{F}, \delta \mathcal{P}]^{3}\right)=\operatorname{Tr}(\mathcal{F}) \operatorname{det}([\mathcal{F}, \delta \mathcal{P}]) .
\end{aligned}
$$

where the proportionality constant is just the flavor diagonal product of Green's functions. Similar contributions arise from the other terms in (71) and all in all we obtain the expected result $\Delta_{\mathrm{CP}} \propto \operatorname{Tr}(\mathcal{F}) \operatorname{det}([\mathcal{F}, \delta \mathcal{P}])$. 


\section{Non-Perturbative Calculations of the Reflection and Trans- mission Coefficients}

In this part we calculate the reflection and transmission coefficients of the fermions in the nonperturbative regime (large Yukawa couplings). This is possible at the expense of working within a single generation framework. Thus CPV is absent and therefore thermal effects, in particular thermal masses, are neglected for simplicity. Our rationale here is that our results below, for the reflection coefficients in the single flavor case, would also hold when promoting the model to three generations [19]. The main difference is the appearance of CPV phases in the thermal masses [see e.g. eq. (27)]. These are expected to be only mildly suppressed by an order one factor $[1 / 8(1 / 32)$ for the quark singlets(doublets) in the 4D SM case] which we shall take into account in our final estimation for the resultant $\mathrm{BAU}^{2}$. Consequently, below we shall assume the following dispersion relations

$$
E=\sqrt{m_{n}^{2}+k_{3}^{2}}
$$

where here and below, in order not to confuse with the perturbative approach, we use $m_{n}=p_{5}^{(n)}$.

As before, for $x_{3}<0$ the system is in the unbroken phase while for $x_{3}>x_{3}^{0}$ the system is in the broken phase. In order to calculate the reflection and transmission coefficients we solve the Dirac equations. for the two regions separately and sew the solutions at $x_{3}=0$. As mentioned before the spin up and down states do not mix in the presence of the localizer's VEV and thus below we only consider the wave function for the spin down states where similar derivation can be applied to the spin up case.

\section{D.1 Unbroken Phase}

For $x^{3}<0$, the relevant positive frequency solutions are described by plane waves propagating in the positive $x^{3}$ direction with momenta $k_{3}$. Since we are dealing with $T \sim R^{-1}$ we will mostly be interested in the dynamic of the lowest KK states. For each representation this implies a single zero mode which can be either incoming or outgoing, but not both. For simplicity we only consider particle with energies less than $2 / R$ and therefore in addition to the zero-mode, a massive Dirac state corresponding to the first KK level can be on shell (while higher KK modes are Boltzmann suppressed). Consequently we also consider incoming or outgoing massive states.

\footnotetext{
${ }^{2}$ Note that in [19], CP violation originated from a different source, namely twisting [22, 23], so that the above mild suppression is absent and an asymmetry was induced even in the absence of these thermal effects.
} 
From (18,2644,48) we find that the solution is characterized by a KK number $n$ (considering as an example an incoming spin-up fermion):

$$
\chi\left(x^{3}<0, n, k_{3}\right)=N_{\chi}^{\mathrm{u}} e^{i\left(k_{3} x^{3}-E t\right)}\left(\begin{array}{l}
\cos \left(n \frac{x^{5}}{R}\right) \sqrt{E+k_{3}} \\
\sin \left(n \frac{x^{5}}{R}\right) \sqrt{E-k_{3}}
\end{array}\right)
$$

where $N_{\chi}^{\mathrm{u}}$ is a normalization constants and

$$
E=\sqrt{m_{n}^{2}+k_{3}^{2}}, \quad m_{n}^{2}=\frac{n^{2}}{R^{2}}
$$

Similar expression would hold for the outgoing (reflected) particles which can be obtained from the above via changing $k_{3}$ to $-k_{3}$. As before, we see that the reflected states do not contain a zero mode for the spin-up fermions. Similarly, for spin-down fermions there is no incoming zero mode.

We can now extract the reflection coefficients at $T \sim 1 / R$ by considering an incoming zero mode with $E \gtrsim 1 / R$ (to allow for an on shell reflected state) which is reflected to a KK tower of massive states. Thus in the unbroken phase the wave function of the fermions is given by

$$
\begin{aligned}
\Psi^{\downarrow}\left(x^{3}<0\right)=\quad & e^{-i E_{0} t}\left[\sum_{n} R_{n} e^{-i k_{3}^{n} x^{3}}\left(\begin{array}{c}
\cos \left(\frac{n x^{5}}{R}\right) \sqrt{\frac{E-k_{3}^{n}}{\pi R}} \\
\sin \left(\frac{n x^{5}}{R}\right) \sqrt{\frac{E+k_{3}^{n}}{\pi R}}
\end{array}\right)+I_{0} e^{i E x^{3}} \sqrt{\frac{E}{\pi R}}\left(\begin{array}{l}
1 \\
0
\end{array}\right)\right. \\
& \left.+I_{1} e^{i k_{3}^{1} x^{3}}\left(\begin{array}{l}
\cos \left(\frac{x^{5}}{R}\right) \sqrt{\frac{E+k_{3}^{1}}{\pi R}} \\
\sin \left(\frac{x^{5}}{R}\right) \sqrt{\frac{E-k_{3}^{1}}{\pi R}}
\end{array}\right)\right],
\end{aligned}
$$

where above and below we use normalized wave functions for the part which depends on $x^{5}$, we set the normalization constant for the incoming particle to one and unless otherwise specified the summation of $n$ is implied and taken from one to the highest KK mode. As mentioned above we only consider incoming modes with $E<2 / R$. Thus one can distinguish between two cases: (i) the incoming mode is a zero mode, which implies $I_{0}=1$ and $I_{1}=0$, or (ii) the incoming mode is a first KK mode which implies setting $I_{1}=1$ and $I_{0}=0$.

\section{D.2 Broken Phase}

For $x^{3}>0$ the general solution depends on $f\left(x^{3}, x^{5}\right)$ and cannot be found analytically. Recall that, for simplicity, we adopt the thin wall approximation (in the $x^{3}$ direction) and assume a step function (in the $x^{5}$ direction) for the localizer, eq. (19). To simplify our notations below we use:

$$
f_{i j}\left(x^{3}, x^{5}\right)=\left\{\begin{array}{ll}
0 & x^{3} \leq 0 \\
f & x^{3}>0
\end{array} .\right.
$$


Thus, for a given KK mode, the positive frequency solution is given by:

$$
\chi\left(x^{3}>0, n, k_{3}^{\prime}\right)=N_{\chi n}^{\mathrm{b}} e^{i\left(k_{3}^{\prime} x^{3}-E t\right)}\left(\begin{array}{l}
u_{n}^{+}\left(x^{5}\right) \sqrt{E+k_{3}^{\prime}} \\
u_{n}^{-}\left(x^{5}\right) \sqrt{E-k_{3}^{\prime}}
\end{array}\right)
$$

where in this case $k_{3}^{\prime 2}$, the square of the momentum in the $x^{3}$ direction, can be either negative or positive, corresponding to on shell and virtual modes respectively. Solving for the $x^{5}$ dependent part, using eqs. (18|2644|48), we find that there are two types of solutions: an exponential solution corresponding to a zero mode,

$$
u_{0}^{+}\left(x^{5}\right)=\sqrt{\frac{f}{e^{2 \pi f R}-1}} e^{f x^{5}}, \quad u_{0}^{-}\left(x^{5}\right)=0, \quad E^{2}=k_{3}^{\prime 2}=k_{3}^{2}
$$

and an oscillatory solution which corresponds to higher KK modes,

$$
\begin{aligned}
& u_{n}^{+}\left(x^{5}\right)=\sqrt{\frac{n^{2}}{\pi R\left(f^{2} R^{2}+n^{2}\right)}} \cdot\left[\cos \left(k_{5}^{n} x^{5}\right)+\frac{f}{k_{5}^{n}} \sin \left(k_{5}^{n} x^{5}\right)\right], \\
& u_{n}^{-}\left(x^{5}\right)=\sqrt{\frac{1}{\pi R}} \cdot \sin \left(k_{5}^{n} x^{5}\right), \quad E^{2}=k_{3}^{\prime 2}+k_{5}^{n 2}=k_{3}^{\prime 2}+f^{2}+\frac{n^{2}}{R^{2}} .
\end{aligned}
$$

Hence in the broken phase the wave function of an outgoing fermion with positive spin is given by

$$
\begin{aligned}
& \chi\left(x^{3}>0\right)=e^{-i E t}\left\{\sum_{n} T_{n} e^{-i k_{3}^{\prime n} x^{3}}\left(\begin{array}{c}
{\left[\cos \left(\frac{n x^{5}}{R}\right)+\frac{f}{k_{5}^{n}} \sin \left(\frac{n x^{5}}{R}\right)\right] \sqrt{\frac{E+k_{3}^{\prime n}}{\pi R}} \sqrt{\frac{n^{2}}{n^{2}+f^{2} R^{2}}}} \\
\sin \left(\frac{n x^{5}}{R}\right) \sqrt{\frac{E-k_{3}^{\prime n}}{\pi R}}
\end{array}\right),\right. \\
& \left.+T_{0} e^{i E x^{3}+f x^{5}} \sqrt{\frac{2 E f}{e^{2 \pi f R}-1}}\left(\begin{array}{l}
1 \\
0
\end{array}\right)\right\}
\end{aligned}
$$

where

$$
k_{3}^{\prime n}=\sqrt{E^{2}-m_{n}^{2}}=\sqrt{E^{2}-f^{2}-\frac{n^{2}}{R^{2}}} .
$$

It is important to note that since in our framework $f R$ is large (to produce significant localization), $f R \sim 5-10$, the non-zero modes in the broken phase are very heavy. Thus only the zero mode is produced on-shell and be transmitted through from the unbroken phase.

\section{D.3 Computation of the Reflection and Transmission Coefficients}

We can equate the expressions for the wave functions in the broken and unbroken phase, and extract the reflection and transmission coefficients. Below we distinguish between the two possibilities for an incoming state, namely between an incoming zero mode and an incoming first KK mode. As a consistency check we also verify below that our solutions make sense in the $f \rightarrow 0$ limit which can be computed analytically. 


\section{D.3.1 Reflection Coefficients for an Incoming Zero Mode}

The reflection and transmission coefficients are found by requiring that the wave function is continuous at $x^{3}=0$. This is done by comparing the expressions in (76) and (81). We note that the functional dependence on $x^{5}$ for the lower component (negative helicity) in both cases is equal up to a kinematic factor. This implies the following relation between the coefficients:

$$
\frac{T_{n}^{2}}{R_{n}^{2}}=\frac{E+k_{3}^{n}}{E-k_{3}^{\prime n}}=\frac{E+\sqrt{E^{2}-\frac{n^{2}}{R^{2}}}}{E-\sqrt{E_{0}^{2}-f^{2}-\frac{n^{2}}{R^{2}}}} .
$$

Substituting the above relation into the upper component of (76) 81) we get the following equation for the reflection coefficients (after multiplying both sides by $\sqrt{\frac{\pi R}{E}}$ ):

$$
\begin{aligned}
1+\sum_{n} R_{n} e_{n} \cos (n w)= & \sqrt{\frac{2 \pi Y}{e^{2 \pi Y}-1}} T_{0} e^{w Y}+ \\
& \sum_{n} R_{n} e_{n} A_{n}\left[\cos (n w)+\frac{Y}{n} \sin (n w)\right]
\end{aligned}
$$

where

$$
\begin{aligned}
w & \equiv x^{5} / R, \quad X \equiv E R, \quad Y \equiv f R \\
A_{n} & \equiv \sqrt{\frac{n^{2}}{Y^{2}+n^{2}}} \cdot \sqrt{\frac{E+k_{3}^{n}}{E-k_{3}^{n}}} \cdot \sqrt{\frac{E+k_{3}^{\prime n}}{E-k_{3}^{\prime n}}}, \quad e_{n} \equiv \sqrt{1-\frac{k_{3}^{n}}{E}} \quad n \neq 0 .
\end{aligned}
$$

In order to find the values of the various coefficients we expand all functions in the cosine basis,

$$
\begin{aligned}
e^{w Y} & =\frac{1}{\pi} \cdot \frac{e^{Y \pi}-1}{Y}+\frac{2 Y}{\pi} \sum_{n} \frac{(-1)^{n} e^{Y \pi}-1}{Y^{2}+n^{2}} \cos (n w), \\
\sin (n w) & =\frac{1}{\pi} \cdot \frac{1-(-1)^{n}}{n}+\frac{2 n}{\pi} \sum_{m} \frac{1-(-1)^{n+m}}{n^{2}-m^{2}} \cos (m w), \quad m \neq n
\end{aligned}
$$

where in the second line if $m=n$ the coefficient is zero. Substitute (86) into (84) and collecting terms we get:

$$
\begin{aligned}
& 1-\sqrt{\frac{2}{\pi Y}} \cdot T_{0} \cdot \sqrt{\frac{e^{Y \pi}-1}{e^{Y \pi}+1}}-\frac{Y}{\pi} \cdot \sum_{n} R_{n} e_{n} A_{n} \cdot \frac{1-(-1)^{n}}{n^{2}} \\
& +\sum_{n m}\left\{R_{n} e_{n}\left[\delta_{n m}-A_{n} \cdot\left(\delta_{n m}+\frac{2 Y}{\pi} \cdot \frac{1-(-1)^{n+m}}{n^{2}-m^{2}}\left(1-\delta_{n m}\right)\right)\right]\right. \\
& \left.-\quad N_{\chi 0}^{\mathrm{b}} \sqrt{\frac{2 Y}{\pi}} \cdot \frac{2 Y}{Y^{2}+n^{2}} \cdot \frac{(-1)^{n} e^{Y \pi}-1}{\sqrt{e^{2 Y \pi}-1}} \delta_{n m}\right\} \cos (m w)=0
\end{aligned}
$$


where summation over $n, m$ begins at one.

We can use the relation for the zeroth element to express $T_{0}$ as a function all the other unknowns,

$$
T_{0}=\sqrt{\frac{Y \pi}{2} \cdot \frac{e^{Y \pi}+1}{e^{Y \pi}-1}}\left[1-\frac{Y}{\pi} \sum_{n} R_{n} e_{n} A_{n} \cdot \frac{1-(-1)^{n}}{n^{2}}\right] .
$$

Substituting (88) back into (87) we get,

$$
\begin{aligned}
& \sum_{n m} R_{n} e_{n}\left\{\delta_{n m}-A_{n}\left[\delta_{n m}+\frac{2 Y}{\pi} \cdot \frac{1-(-1)^{n+m}}{n^{2}-m^{2}}\left(1-\delta_{n m}\right)\right.\right. \\
- & \left.\left.\frac{1-(-1)^{n}}{\pi n^{2}} \cdot \frac{2 Y^{3}}{Y^{2}+m^{2}} \cdot \frac{(-1)^{m} e^{Y \pi}-1}{e^{Y \pi}-1}\right]\right\} \cos (m w) \\
= & \frac{2 Y^{2}}{e^{Y \pi}-1} \sum_{m} \frac{(-1)^{m} e^{Y \pi}-1}{Y^{2}+m^{2}} \cdot \cos (m w) .
\end{aligned}
$$

Let us write the above relation in a matrix form, describing the set of equations for the various Fourier coefficients

$$
M_{m n}^{R_{n}} R_{n}=V_{n}
$$

where $M_{m n}^{R_{n}}$ and $V_{n}$ can be directly extracted from (89). Inverting $M$ yields the solution for the reflection coefficients

$$
R_{n}=\left(M_{m n}^{R_{n}}\right)^{-1} V_{n}
$$

This of course is done numerically.

\section{D.3.2 Reflection Coefficients for an Incoming First KK Mode with Spin Up}

As before, we write the relation between the reflection and transmission coefficients, this time for higher KK modes,

$$
T_{n}=\left\{\begin{array}{ll}
R_{n} \sqrt{\frac{E_{0}+k_{3}^{n}}{E_{0}-k_{3}^{\prime n}}} & n \neq 1 \\
R_{n} \sqrt{\frac{E_{0}+k_{3}^{n}}{E_{0}-k_{3}^{\prime n}}}+\sqrt{\frac{E_{0}-k_{3}^{n}}{E_{0}-k_{3}^{\prime n}}} & n=1
\end{array} .\right.
$$

Again we substitute the above to get,

$$
\begin{aligned}
& \sqrt{1+\frac{k_{3}^{1}}{E}} \cos w+\sum_{n} R_{n} e_{n} \cos (n w)=\sqrt{\frac{2 \pi Y}{e^{2 \pi Y}-1}} T_{0} e^{w Y}+ \\
& \sum_{n} e_{n}\left(R_{n} A_{n}+B_{n} \delta_{1 n}\right)\left[\cos (n w)+\frac{Y}{n} \sin (n w)\right]
\end{aligned}
$$


where

$$
B_{n} \equiv \sqrt{\frac{n^{2}}{Y^{2}+n^{2}}} \cdot \sqrt{\frac{E+k_{3}^{\prime n}}{E-k_{3}^{\prime n}}}
$$

Expanding and collecting terms, using (86), one obtains,

$$
\begin{aligned}
& -\sqrt{\frac{2}{\pi Y}} \cdot T_{0} \cdot \sqrt{\frac{e^{Y \pi}-1}{e^{Y \pi}+1}}-\frac{Y}{\pi} \cdot \sum_{n} e_{n} R_{n} A_{n} \cdot \frac{1-(-1)^{n}}{n^{2}}-\frac{2 Y}{\pi} e_{1} B_{1} \\
+ & \sum_{n m}\left\{R_{n} e_{n}\left[\delta_{n m}-A_{n} \cdot\left(\delta_{n m}+\frac{2 Y}{\pi} \cdot \frac{1-(-1)^{n+m}}{n^{2}-m^{2}}\left(1-\delta_{n m}\right)\right)\right]\right. \\
- & \left.T_{0} \sqrt{\frac{2 Y}{\pi}} \cdot \frac{2 Y}{Y^{2}+n^{2}} \cdot \frac{(-1)^{n} e^{Y \pi}-1}{\sqrt{e^{2 Y \pi}-1}} \delta_{n m}\right\} \cos (m w) \\
= & \left(e_{1} B_{1}-\sqrt{1+\frac{k_{3}^{1}}{E}}\right) \cdot \cos w+e_{1} B_{1} \sum_{m=2} \frac{2 Y}{\pi} \cdot \frac{1+(-1)^{m}}{1-m^{2}} \cdot \cos (m w)
\end{aligned}
$$

which leads to,

$$
T_{0}=-\sqrt{\frac{Y \pi}{2} \cdot \frac{e^{Y \pi}+1}{e^{Y \pi}-1}}\left[\frac{2 Y}{\pi} e_{1} B_{1}+\frac{Y}{\pi} \sum_{n} R_{n} e_{n} A_{n} \cdot \frac{1-(-1)^{n}}{n^{2}}\right] .
$$

Substituting (96) in (95) we get,

$$
\begin{aligned}
& \sum_{n m} R_{n} e_{n}\left\{\delta_{n m}-A_{n}\left[\delta_{n m}+\frac{2 Y}{\pi} \cdot \frac{1-(-1)^{n+m}}{n^{2}-m^{2}}\left(1-\delta_{n m}\right)\right.\right. \\
- & \left.\left.\frac{1-(-1)^{n}}{\pi n^{2}} \cdot \frac{2 Y^{3}}{Y^{2}+m^{2}} \cdot \frac{(-1)^{m} e^{Y \pi}-1}{e^{Y \pi}-1}\right]\right\} \cos (m u)=\left(e_{1} B_{1}-\sqrt{1+\frac{k_{3}^{1}}{E}}\right) \cos u \\
+ & e_{1} B_{1} \frac{2 Y}{\pi} \sum_{m}\left[\frac{1+(-1)^{m}}{1-m^{2}}\left(1-\delta_{1 m}\right)-\frac{2 Y^{2}}{Y^{2}+m^{2}} \cdot \frac{(-1)^{m} e^{Y \pi}-1}{e^{Y \pi}-1}\right] \cos (m u),
\end{aligned}
$$

which may again be used to extract the reflection coefficients.

\section{D.3.3 Reflection Coefficients for an Incoming First KK Mode with Spin Down}

This time,

$$
T_{n}= \begin{cases}R_{n} \sqrt{\frac{E_{0}-k_{3}^{n}}{E_{0}+k_{3}^{\prime n}}}, & n \neq 1 \\ R_{n} \sqrt{\frac{E_{0}-k_{3}^{n}}{E_{0}+k_{3}^{\prime n}}}+\sqrt{\frac{E_{0}+k_{3}^{n}}{E_{0}+k_{3}^{\prime n}}}, & n=1\end{cases}
$$


which, after substitution, gives,

$$
\begin{aligned}
& R_{0}+\sqrt{1-\frac{k_{3}^{1}}{E}} \cos w+\sum_{n} N_{\mathrm{Rn}}^{\mathrm{u}} e_{n}^{\prime} \cos (n w)= \\
& \sum_{n} e_{n}^{\prime}\left(R_{n} A_{n}^{\prime}+B_{n}^{\prime} \delta_{1 n}\right)\left[\cos (n w)+\frac{Y}{n} \sin (n w)\right],
\end{aligned}
$$

with

$$
\begin{aligned}
A_{n}^{\prime} & \equiv \sqrt{\frac{n^{2}}{Y^{2}+n^{2}}} \cdot \sqrt{\frac{E-k_{3}^{n}}{E+k_{3}^{n}}} \cdot \sqrt{\frac{E-k_{3}^{\prime n}}{E+k_{3}^{\prime n}}}, \quad e_{n}^{\prime} \equiv \sqrt{1+\frac{k_{3}^{n}}{E}} \\
B_{n}^{\prime} & \equiv \sqrt{\frac{n^{2}}{Y^{2}+n^{2}}} \cdot \sqrt{\frac{E-k_{3}^{\prime n}}{E+k_{3}^{\prime n}}} .
\end{aligned}
$$

Finally, repeating the same procedure as before, one gets,

$$
\begin{aligned}
& R_{0}-\frac{Y}{\pi} \cdot \sum_{n} e_{n}^{\prime} N_{\mathrm{Rn}}^{\mathrm{u}} A_{n}^{\prime} \cdot \frac{1-(-1)^{n}}{n^{2}}-\frac{2 Y}{\pi} e_{1}^{\prime} B_{1}^{\prime} \\
+ & \sum_{n m}\left\{N_{\mathrm{Rn}}^{\mathrm{u}} e_{n}^{\prime}\left[\delta_{n m}-A_{n}^{\prime} \cdot\left(\delta_{n m}+\frac{2 Y}{\pi} \cdot \frac{1-(-1)^{n+m}}{n^{2}-m^{2}}\left(1-\delta_{n m}\right)\right)\right]\right\} \cos (m w) \\
= & \left(e_{1}^{\prime} B_{1}^{\prime}-\sqrt{1-\frac{k_{3}^{1}}{E}}\right) \cdot \cos w+e_{1}^{\prime} B_{1}^{\prime} \sum_{m=2} \frac{2 Y}{\pi} \cdot \frac{1+(-1)^{m}}{1-m^{2}} \cdot \cos (m w) .
\end{aligned}
$$

\section{D.3.4 Check $Y \rightarrow 0$}

In order to obtain some intuition and check our results, let us check the above in the small energy, small barrier limit (see (85)),

$$
Y \rightarrow 0
$$

In that limit, to leading order, relation (89) is simplified

$$
\sum_{n m} R_{n} e_{n}\left(A_{n}-1\right) \delta_{m n} \cos (m w)=\frac{2 Y}{\pi} \sum_{m} \frac{1-(-1)^{m}}{m^{2}} \cos (m w),
$$

and we find the following expressions for $A_{n}$ (85),

$$
A_{n}=-1+2 \frac{X}{n} \sqrt{\frac{X^{2}}{n^{2}}-1}+2 \frac{X^{2}}{n^{2}}+\mathcal{O}\left(Y^{2}\right) .
$$

Under the above approximation the explicit matrix $M_{m n}$ (90) can easily be inverted and 
we find the following approximated solution for the reflection coefficients: ${ }^{3}$

$$
R_{2 n-1}=\frac{4 Y}{\pi\left(A_{2 n-1}-1\right) e_{2 n-1}(2 n-1)^{2}}, \quad R_{2 n}=0 .
$$

Furthermore, using (88) we also have the transmission coefficient,

$$
T_{0}=\left[1+\mathcal{O}\left(Y^{2}\right)\right]\left[1-\frac{8 Y^{2}}{\pi^{2}} \sum_{2 n-1} \cdot \frac{A_{2 n-1}}{\left(A_{2 n-1}-1\right)(2 n-1)^{4}}\right] .
$$

The above simplifications and the generalizations to the reflections of other modes, allow us to apply several checks for our numerical results.

- Vanishing of odd reflection coefficients for small $Y$. Verified numerically.

- When $X \leq 1$ we expect $N_{\chi 0}^{\mathrm{b}}$ to be one. Verified numerically.

- For $2 \geq X \geq 1$ and $Y \ll 1$ we expect $N_{\chi 0}^{\mathrm{b}}$ to deviate from unity only at the order of $Y^{2}$. The reflection coefficient of the first KK mode, is expected to deviate at order $Y$. Verified numerically.

Another way to verify that our numerical results is correct is to check that it is satisfying the unitarity condition as we discuss next.

\section{E Unitarity Conditions}

We now wish to establish the unitarity conditions for our five dimensional scattering process. To obtain intuition, we consider the relation between the reflection and transmission coefficient in the following two cases: (i) a non-relativistic scalar - one particle quantum mechanics described via Schroedinger equation and (ii) relativistic spin half fermion - one particle quantum mechanics described via Dirac equation.

\section{E.1 Schroedinger Equation}

We wish to calculate the reflection of transmission coefficients of a particle in a step function potential:

$$
V(x)=V \theta\left(x^{3}\right)
$$

\footnotetext{
${ }^{3}$ The reason that the even coefficients vanish is probably related to fact that the potential is symmetric with respect to the reflection around the point $w=\pi / 2$. Thus to first order only transitions between states that respect this symmetry are allowed.
} 
The Schroedinger equation reads:

$$
\left(-\nabla^{2}+V(x)\right) \psi=E \psi
$$

For an incoming particle with momentum $\vec{k}=(0,0, k)$ the solution is given by

$$
\psi\left(x^{3}\right)=\theta\left(-x^{3}\right)\left[e^{i k x^{3}}+r e^{-i k x^{3}}\right]+\theta\left(x^{3}\right) t e^{i k^{\prime} x^{3}} \quad k^{\prime 2}=E-V .
$$

Continuity of the wave function and its derivative requires,

$$
r=\frac{1-R_{k}}{1+R_{k}}, \quad t=\frac{2}{1+R_{k}}, \quad R_{k}=\frac{k^{\prime}}{k}
$$

To obtain the unitarity condition, we consider the Schroedinger conserved current equation (for static density all over space) which is given by

$$
\vec{\nabla} \cdot\left(\psi^{*} \vec{\nabla} \psi-\psi \vec{\nabla} \psi^{*}\right)=0
$$

Integrating the above from $-\infty$ to $\infty$ and omitting the interference term we find

$$
\left.\left(\psi^{*} \vec{\nabla} \psi-\psi \vec{\nabla} \psi^{*}\right)\right|_{-\infty} ^{\infty}=0,
$$

which implies for (109) the relation:

$$
|r|^{2}+R_{k}|t|^{2}=1
$$

and clearly (110) is consistent with the above.

\section{E.2 Dirac Equation - 4D Case}

Let us first consider a reflection problem from a one dimensional wall embedded in four dimensions. The Dirac equation reads,

$$
\gamma^{0}[\nabla \cdot \vec{\gamma}+V(x)] \psi=E \psi
$$

For a massless incoming spin down particle with momentum $k^{\mu}=(k, 0,0, k)$ the solution is (in the chiral basis),

$$
\begin{aligned}
\psi\left(x^{3}\right) & =\theta\left(-x^{3}\right)\left[e^{i k x^{3}}\left(\begin{array}{c}
0 \\
\sqrt{2 E} \\
0 \\
0
\end{array}\right)+r e^{-i k x^{3}}\left(\begin{array}{c}
0 \\
0 \\
0 \\
\sqrt{2 E}
\end{array}\right)\right]+\theta\left(x^{3}\right) t e^{i k^{\prime} x^{3}}\left(\begin{array}{c}
0 \\
\sqrt{E+k^{\prime}} \\
0 \\
\sqrt{E-k^{\prime}}
\end{array}\right) \\
k^{\prime 2} & =E^{2}-V^{2} .
\end{aligned}
$$


Note that in this case (which is different from the $5 \mathrm{D}$ case) the transmitted particle is massive while the reflected one is massless. From continuouity of the wave function one finds:

$$
r=\sqrt{\frac{1-R_{k}}{1+R_{k}}}, \quad t=\sqrt{\frac{2}{1+R_{k}}}, \quad R_{k}=\frac{k^{\prime}}{k} .
$$

Again, unitarity condition is found through the Dirac conserved current equation,

$$
\vec{\nabla} \cdot(\bar{\psi} \vec{\gamma} \psi)=0
$$

which gives

$$
\left.(\bar{\psi} \vec{\gamma} \psi)\right|_{-\infty} ^{\infty}=0
$$

We thus obtain the condition,

$$
|r|^{2}+R_{k}|t|^{2}=1
$$

\section{E.3 Dirac Equation - 5D Case}

We now move on to discuss the $5 \mathrm{D}$ problem. For finite temperature of order $(1 / R)$ we assume it is enough to consider only the zero modes and the first KK state.

\section{E.3.1 Incoming Spin-Down Massless Particle}

Thus we will assume that the incoming particle has energy slightly above $(1 / R)$ and that it is reflected into an on shell first excited KK state while the on shell transmitted particle is massless. The 5D Dirac equation reads

$$
\gamma^{0}\left[\nabla \cdot \vec{\gamma}+V(x)+\gamma_{5} \partial_{5}\right] \psi=E \psi
$$

For a massless incoming particle with momentum $k^{\mu}=(k, 0,0, k)$ the solution is (again in the chiral basis)

$$
\begin{aligned}
\psi\left(x^{3}\right) & =\theta\left(-x^{3}\right)\left[e^{i k x^{3}}\left(\begin{array}{c}
0 \\
\sqrt{2 E} \\
0 \\
0
\end{array}\right)+r e^{-i k^{\prime} x^{3}}\left(\begin{array}{c}
0 \\
\sqrt{E-k^{\prime}} \\
0 \\
\sqrt{E+k^{\prime}}
\end{array}\right)\right]+\theta\left(x^{3}\right) t e^{i k x^{3}}\left(\begin{array}{c}
0 \\
\sqrt{2 E} \\
0 \\
0
\end{array}\right) \\
k^{\prime 2} & =E^{2}-\frac{1}{R^{2}} .
\end{aligned}
$$


Since the extra dimension is compact, there is no current flowing in this direction. The conserved current is therefore

$$
\vec{\nabla} \cdot\left(\bar{\psi}^{*} \vec{\gamma} \psi\right)=0
$$

which implies,

$$
R_{k}|r|^{2}+|t|^{2}=1
$$

\section{E.3.2 Incoming Spin-Down First KK Mode}

For a massive incoming particle with momentum $k^{\mu}=\left(k, 0,0, k^{\prime}\right)$ the solution this time is

$$
\begin{aligned}
\psi\left(x^{3}\right) & =\theta\left(-x^{3}\right)\left[e^{i k^{\prime} x^{3}}\left(\begin{array}{c}
0 \\
\sqrt{E+k^{\prime}} \\
0 \\
\sqrt{E-k^{\prime}}
\end{array}\right)+r e^{-i k^{\prime} x^{3}}\left(\begin{array}{c}
0 \\
\sqrt{E-k^{\prime}} \\
0 \\
\sqrt{E+k^{\prime}}
\end{array}\right)\right]+\theta\left(x^{3}\right) t e^{i k x^{3}}\left(\begin{array}{c}
0 \\
\sqrt{2 E} \\
0 \\
0
\end{array}\right) \\
k^{\prime 2} & =E^{2}-\frac{1}{R^{2}} .
\end{aligned}
$$

As before, one obtains the relation

$$
|r|^{2}+R_{k}^{-1}|t|^{2}=1
$$

\section{E.3.3 Incoming Spin-Up First KK Mode}

Here the solution is

$$
\begin{aligned}
\psi\left(x^{3}\right) & =\theta\left(-x^{3}\right)\left[e^{i k^{\prime} x^{3}}\left(\begin{array}{c}
\sqrt{E-k^{\prime}} \\
0 \\
\sqrt{E+k^{\prime}} \\
0
\end{array}\right)+r_{1} e^{-i k^{\prime} x^{3}}\left(\begin{array}{c}
\sqrt{E+k^{\prime}} \\
0 \\
\sqrt{E-k^{\prime}} \\
0
\end{array}\right)\right]+\theta\left(-x^{3}\right) r_{0} e^{-i k x^{3}}\left(\begin{array}{c}
\sqrt{2 E} \\
0 \\
0 \\
0
\end{array}\right) \\
k^{\prime 2} & =E^{2}-\frac{1}{R^{2}}
\end{aligned}
$$

and therefore the unitarity condition

$$
\left|r_{1}\right|^{2}+R_{k}^{-1}\left|r_{0}\right|^{2}=1
$$

